\title{
Mathematical Analysis of a Bohr Atom Model
}

\author{
Goong Chen ${ }^{1,2}$, Zhonghai Ding ${ }^{3}$, Sze-Bi Hsu ${ }^{1,4}$, Moochan Kim², \\ and Jianxin $\mathrm{Zhou}^{1}$
}

\begin{abstract}
N. Bohr proposed in 1913 a model for atoms and molecules by synthesizing Planck's quantum hypothesis with classical mechanics. When the atom number $Z$ is small, his model provides good accuracy for the ground state energy. When $Z$ is large, his model is not as accurate but still provides a good trend agreeing with the experimental values of the ground state energy of atoms.

The main objective of this paper is to provide a rigorous mathematical analysis for the Bohr atom model. We have established the following:

(1) An existence proof of the global minimizer of the ground state energy through scaling;

(2) A careful study of the critical points of the energy function. Such critical points include both the stable steady-state electron configurations as well as unstable saddle-type configurations.

(3) Coplanarity of certain electron configurations.
\end{abstract}

Numerical examples and graphics are also illustrated.

1: Department of Mathematics, Texas A\&M University, College Station, TX 77843

2: Institute for Quantum Studies and Department of Physics, Texas A\&M University, College Station, TX 77843

3: Department of Mathematical Sciences, University of Nevada, Las Vegas, NV 89154

4: Department of Mathematics, National Tsing Hua University, Hsinchu, Taiwan, R.O.C. Work completed while on sabbatical leave at Texas A\&M University. Supported in part by an NSC grant of R.O.C. 


\section{Introduction}

In 1913, N. Bohr published a series of three papers $[1,2,3]$ describing his approach for modeling atoms and molecules by synthesizing Planck's quantum hypothesis with classical mechanics. Bohr tried to explain the hydrogen spectral lines with a radical "planetary" model of electrons orbiting around a nucleus. He made a set of assumptions to quantify his model, leading to the existence in the atom a discrete set of stable, stationary orbits for electrons:

(1) The dynamical equilibrium of the stationary orbits is achieved by balancing the electrostatic Coulomb forces of attraction against the centrifugal effect and the interelectronic repelling of the orbital motion in classical mechanics.

(2) Stationary states satisfy the quantization condition that the ratio of the total kinetic energy of the electron to its orbital frequency be an integral multiple of $\pi \hbar$. For circular orbits, this signifies that the angular momentum of the electron is restricted to integral multiples of $\hbar$.

(3) Energy is emitted only when an electron makes a "jump" (i.e., non-continuous) transition between two stationary orbits, and the frequency of such a radiation emission is determined by $\Delta E / 2 \pi \hbar$, where $\Delta E$ is the energy difference between the two orbits where the transition occurs.

From now on, vectors will be denoted by bold letters. For the hydrogen atom, Bohr's assumptions work as follows. The total energy of the electron on a circular orbit with radius $r$ and velocity $\boldsymbol{v}$ is

$$
\begin{aligned}
E & =\text { kinetic energy }+ \text { potential energy } \\
& =\frac{m_{e} v^{2}}{2}-\frac{Z e^{2}}{r}
\end{aligned}
$$

where

$$
\begin{aligned}
m_{e} & =\text { the mass of the electron, } \\
e & =\text { the charge of the electron, } \\
Z e & =\text { the positive charge of the nucleus. }
\end{aligned}
$$

Since

$$
\begin{aligned}
\boldsymbol{L} & =\text { the angular momentum } \\
& =\boldsymbol{r} \times \boldsymbol{p} \quad\left(\boldsymbol{p} \equiv \text { the linear momentum }=m_{e} \boldsymbol{v}\right), \\
|\boldsymbol{L}| & =L=m_{e} v r
\end{aligned}
$$

which, in turn, by Bohr's quantization assumption, satisfies

$$
L=n \hbar .
$$

From (1.1)-(1.3), we now have

$$
\begin{aligned}
E & =\frac{m_{e}^{2} v^{2} r^{2}}{2 m_{e} r^{2}}-\frac{Z e^{2}}{r} \\
& =\frac{L^{2}}{2 m_{e} r^{2}}-\frac{Z e^{2}}{r} \\
& =\frac{n^{2} \hbar^{2}}{2 m_{e} r^{2}}-\frac{Z e^{2}}{r} \\
& =\frac{1}{2} \frac{n^{2}}{r^{2}}-\frac{Z}{r}
\end{aligned}
$$


in atomic units (by setting $e=1, \hbar^{2} / m_{e}=1$ ).

\begin{tabular}{|l|c|c|}
\hline Electron numbers $i$ & Electron shells names & Assigned quantum numbers $n_{i}$ \\
\hline $1 \leq i \leq 2$ & $K$ & 1 \\
\hline $3 \leq i \leq 8$ & $L$ & 2 \\
\hline $9 \leq i \leq 18$ & $M$ & 3 \\
\hline $19 \leq i \leq 36$ & $N$ & 4 \\
\hline $37 \leq i \leq 54$ & $O$ & 5 \\
\hline $55 \leq i \leq 86$ & $P$ & 6 \\
\hline $87 \leq i \leq 108$ & $Q$ & 7 \\
\hline
\end{tabular}

Table 1. Assignment of quantum numbers $n_{i}$ for the ground state of an atom with atom number $Z$ from $Z=1$ to 108 .

Minimizing $E=E(r, n, Z)$ with respect to $r$ for $n=1,2,3, \ldots$, for fixed $Z$, we obtain

$$
\widehat{E}_{n} \equiv \min _{r>0} E(r, n, Z)=-\frac{1}{2} \frac{Z^{2}}{n^{2}}, \quad n=1,2,3, \ldots .
$$

These values and their differences totally determine the hydrogen atom's spectral lines.

Sommerfeld later in 1916 generalized Bohr's theory by allowing non-circular orbits and by incorporating relativistic effects, leading to the Bohr-Sommerfeld (old) quantum theory of the hydrogen atom. However, for other atoms, including the simple helium, there are difficulties unaccountable by the Bohr-Sommerfeld theory; see e.g. [6, 7]. Heisenberg worked under both Bohr and Sommerfeld trying to resolve such difficulties, eventually gave up but in the process invented the matrix mechanics during the 1930s.

The objective of the present paper is to analyze, mathematically, a Bohr atom model for the ground-states of general atoms. Such a general Bohr model seems to be well understood by atomic physicists (see, e.g., the pictorials on the website of Patton [11]) but we could not provide an exact citation. The model that we are going to describe below is communicated to us by our colleague, Dr. S.A. Chin [4]. Consider a neutral atom with atom number $Z$. There are $Z$ electrons. The kinetic energy of an electron $i$ moving around a circular orbit of radius $r_{i}$ on the $n$-th shell, $n_{i}=1,2,3, \ldots$, is

$$
\begin{aligned}
T_{i}=-\frac{1}{2} n_{i}^{2} / r_{i}^{2}, \quad & \left(r_{i}=\left|\boldsymbol{r}_{i}\right|, \boldsymbol{r}_{i}=\left(x_{i}, y_{i}, z_{i}\right) \in \mathbb{R}^{3}\right. \text { is the } \\
& \text { position vector of electron } i)
\end{aligned}
$$

For a heuristic derivation of (1.7), see [6, Appendix]. The potential energy is attributed to the Coulomb interactions of electron $j$ with the nucleus and electrons $j$ for $j \neq i$ :

$$
P_{i}=-\frac{Z}{r_{i}}+\sum_{\substack{j \neq i \\ j=1}}^{Z} \frac{1}{r_{i j}} \quad\left(r_{i j}=\left|\boldsymbol{r}_{i}-\boldsymbol{r}_{j}\right|\right) .
$$

Thus the total energy of the atom is

$$
\begin{aligned}
E & =E\left(\boldsymbol{r}_{1}, \boldsymbol{r}_{2}, \ldots, \boldsymbol{r}_{Z}\right)=\sum_{j=1}^{Z}\left(T_{j}+P_{j}\right) \\
& =\sum_{i=1}^{Z}\left(\frac{n_{i}^{2}}{2 r_{i}^{2}}-\frac{Z}{r_{i}}\right)+\sum_{\substack{i, j=1 \\
i \neq j}}^{Z} \frac{1}{2 r_{i j}} .
\end{aligned}
$$


We now pack these $Z$ electrons into the various "electron shells" by the Aufbau Principle ${ }^{1}$ by assigning the values of $n_{i}$ according to Table 1 . These designated integral values of $n_{i}$ give us the Bohr atom model in this paper.

The stable stationary orbit or electron configuration, denoted as $\left(\boldsymbol{r}_{1}^{*}, \boldsymbol{r}_{2}^{*}, \ldots, \boldsymbol{r}_{Z}^{*}\right)$, and the associated ground state energy $E\left(\boldsymbol{r}_{1}^{*}, \boldsymbol{r}_{2}^{*}, \ldots, \boldsymbol{r}_{Z}^{*}\right)$, can now be obtained by

$$
\min _{\boldsymbol{R} \in \mathbb{R}^{3 Z}} E(\boldsymbol{R}) \equiv E\left(\boldsymbol{R}^{*}\right), \quad \boldsymbol{R}^{*} \equiv\left(\boldsymbol{r}_{1}^{*}, \boldsymbol{r}_{2}^{*}, \ldots, \boldsymbol{r}_{Z}^{*}\right) .
$$

In Table 2, we have listed the ground-state energy of all the atoms calculated from (1.10) as well as the known ("exact") experimental values. The reader may find some agreement between these two sets of values, especially when $Z$ is small.

The Bohr model of atoms was derived by Bohr in an ad hoc way at first. The rigorous, wave-mechanical model is the following Schrödinger-Born-Oppenheimer equation describing the many-particle quantum-mechanical behavior

$$
\left(-\frac{1}{2} \sum_{k=1}^{Z} \nabla_{k}^{2}+\frac{1}{2} \sum_{\substack{k, k^{\prime}=1 \\ k \neq k^{\prime}}}^{Z} \frac{1}{r_{k k^{\prime}}}-\sum_{k=1}^{Z} \frac{Z}{r_{k}}\right) \phi(\boldsymbol{R})=E \phi(\boldsymbol{R}) .
$$

As Bohr's model appears to be something of the history, why does it still warrant any attention? The reasons that motivate our study here are three fold:

(i) Recently, through the dimensional scaling ( $D$-scaling) method [8], Svidzinsky, Scully and Hershbach $[12,13]$ have arrived at Bohr's model from the totally quantum-mechanical (SchrödingerBorn-Oppenheimer) model via asymptotics. This has stirred surprise, excitement and interests [15], especially among the researchers in the Institute for Quantum Studies at Texas A\&M and has rekindled efforts in trying to understand the synergism between $D$-scaling, the Schrödinger-Born-Oppenheimer model and the Bohr model for atoms and molecules.

(ii) The Schrödinger-Born-Oppenheimer model (1.11) involves large-scale numerical computation and is rather theoretically intractable, while the Bohr model (1.5), (1.9) and (1.10) requires only desk-top computing, producing outcomes of electron configurations highly valuable and intuitive for atomic experiments and molecular modeling, especially with the incorporation of the Hartree-Fock and other refinement techniques ([6, 7, 5, 10, 14], e.g.).

(iii) Mathematically speaking, even though Bohr's atom model is nearly 90 years old, historically it has not attracted due attention in the mathematics community and, thus, has not undergone rigorous mathematical analysis it rightfully deserves. Many interesting mathematical problems are worth investigation. We hope our mathematical analysis carried out here will improve the understanding of Bohr's atomic model and that of atoms in general at a more fundamental level, with an ultimate goal of improving the modeling and compution of molecules or even building new molecular models.

\section{Existence and Algorithm for the Global Minimizer}

The following problems are of significant mathematical interests, which also have physical importance:

\footnotetext{
${ }^{1}$ The packing of electrons into electron shells by the Aufbau Principle involves also subshells p.d.f, etc. Since here we are only dealing with ground states of atoms, we pack electrons only into the principal $s$ shells.
} 
(i) A rigorous existence proof of ground state energies;

(ii) Stable as well as unstable electron steady-state orbits;

(iii) Geometric configurations: co-planarity and symmetries of electron locations.

We discuss them through a sequence of lemmas and theorems.

From now on, to simplify notation, we often write

$$
\sum_{i=1}^{n} \text { as } \sum_{i}, \sum_{\substack{i, j=1 \\ i \neq j}}^{n} \text { as } \sum_{i \neq j}
$$

unless more clarity of the summation index is deemed necessary.

We begin by letting $Z$ be a positive number and $n$ be a positive integer; $n=1,2,3, \ldots$, define (1) For $n=1$,

$$
E_{1}^{Z}: \mathbb{R}^{3} \rightarrow \mathbb{R}, \quad E_{1}^{Z}\left(\boldsymbol{r}_{1}\right)=\frac{n_{1}^{2}}{2 r_{1}^{2}}-\frac{Z}{r_{1}}, \quad \boldsymbol{r}_{1} \in \mathbb{R}^{3} ;
$$

(2) For $n \geq 2$ and $Z \geq n, E_{n}^{Z}: \mathbb{R}^{3 n} \rightarrow \mathbb{R}$,

$$
E_{n}^{Z}(\mathbf{R})=\sum_{i=1}^{n}\left(\frac{n_{i}^{2}}{2 r_{i}^{2}}-\frac{Z}{r_{i}}\right)+\sum_{\substack{i, j=1 \\ i \neq j}}^{n} \frac{1}{2 r_{i j}}
$$

where $\boldsymbol{R}=\left(\boldsymbol{r}_{1}, \ldots, \boldsymbol{r}_{n}\right), \boldsymbol{r}_{i} \in \mathbb{R}^{3}, r_{i}$ and $r_{i j}$ are defined as in (1.7) and (1.8). The domain of $E_{n}^{Z}$ is then given by $\mathbb{R}^{3 n} \backslash \mathcal{S}_{n}$, where $\mathcal{S}_{n}$ is the singularity manifold of $E_{n}^{Z}$ given by

$$
\mathcal{S}_{n}=\left\{\boldsymbol{R}=\left(\boldsymbol{r}_{1}, \ldots, \boldsymbol{r}_{n}\right) \in \mathbb{R}^{3 n} \mid \boldsymbol{r}_{j}=\mathbf{0} \text { or } \boldsymbol{r}_{j}-\boldsymbol{r}_{k}=0, \text { for some } j, k, 1 \leq j, k \leq n, j \neq k\right\} .
$$

$E_{n}^{Z}$ is obviously in $C^{\infty}\left(\mathbb{R}^{3 n} \backslash \mathcal{S}_{n}\right)$. We are interested in the existence of $\boldsymbol{R}^{*} \in \mathbb{R}^{3 n} \backslash \mathcal{S}_{n}$ such that

$$
E_{n}^{Z}\left(\boldsymbol{R}^{*}\right)=\inf _{\boldsymbol{R} \in \mathbb{R}^{3 n} \backslash \mathcal{S}_{n}} E_{n}^{Z}(\boldsymbol{R}), \quad \text { for } \quad n \geq 2 .
$$

Note that the case of $n=1$ is already solved in (1.6). From now on, we will abbreviate $E_{n}^{Z}$ as $E$ if no ambiguities should arise. Throughout the rest of the section, the reader may find that in all of the proofs given, as long as $n_{i}>0$ for $i=1,2, \ldots, n$, then the proofs go through without any problem, i.e., $n_{i}$ 's do not have to follow the designated values as in Table 1 . We further define

$$
\mathcal{S}_{Z}^{n}=\left\{\boldsymbol{R} \in \mathbb{R}^{3 n} \backslash \mathcal{S}_{n} \mid \sum_{i} \frac{Z}{r_{i}}-\sum_{i \neq j} \frac{1}{2 r_{i j}}>0\right\} .
$$

Lemma 1 (Scaling along a ray). Let $\boldsymbol{R} \in \mathcal{S}_{Z}^{n}$. The function $g: \mathbb{R}_{+} \equiv(0, \infty) \rightarrow \mathbb{R}, g(t) \equiv E(t \boldsymbol{R})$ has a unique global minimum at

$$
t^{*}=t^{*}(\boldsymbol{R})=\arg \min _{t \neq 0, t \in \mathbb{R}} E(t \boldsymbol{R}),
$$

where

$$
\begin{aligned}
& t^{*}>0, g^{\prime}\left(t^{*}\right)=0, \quad g^{\prime \prime}\left(t^{*}\right)>0, \\
& E\left(t^{*} \boldsymbol{R}\right)=-\frac{1}{2} \sum_{i} \frac{n_{i}^{2}}{r_{i}^{2}} \frac{1}{t^{* 2}}=-\frac{1}{2}\left(\sum_{i} \frac{Z}{r_{i}}-\sum_{i \neq j} \frac{1}{r_{i j}}\right)^{2} /\left(\sum_{i} \frac{n_{i}^{2}}{r_{i}^{2}}\right)<0 .
\end{aligned}
$$


Proof. First, we note that $\mathcal{S}_{Z}^{n}$ is a nonempty unbounded open set of $\mathbb{R}^{3 Z}$. It is easy to see that if $\boldsymbol{R} \in \mathcal{S}_{Z}^{n}$, then $t \boldsymbol{R} \in \mathcal{S}_{Z}^{n}$ for any $t \neq 0$, i.e., $\mathcal{S}_{Z}^{n}$ is star-shaped. For $t>0$, we have

$$
\begin{aligned}
& g(t)=\frac{1}{t^{2}} \sum_{i}\left(\frac{n_{i}^{2}}{2 r_{i}^{2}}\right)+\frac{1}{t}\left(-\sum_{i} \frac{Z}{r_{i}}+\sum_{i \neq j} \frac{1}{2 r_{i j}}\right), \\
& g^{\prime}(t)=-\frac{2}{t^{3}} \sum_{i} \frac{n_{i}^{2}}{2 r_{i}^{2}}+\frac{1}{t^{2}}\left(\sum_{i} \frac{Z}{r_{i}}-\sum_{i \neq j} \frac{1}{2 r_{i j}}\right) .
\end{aligned}
$$

Set $g^{\prime}\left(t^{*}\right)=0$ to get the only zero of $g^{\prime}$ at

$$
t^{*}=\frac{\sum_{i} \frac{n_{i}^{2}}{r_{i}^{2}}}{\sum_{i} \frac{Z}{r_{i}}-\sum_{i \neq j} \frac{1}{2 r_{i j}}} .
$$

Note that the denominator in (2.7) is positive since $\boldsymbol{R} \in \mathcal{S}_{Z}^{n}$. So $t^{*}$ is well-defined. Next, we have

$$
\begin{aligned}
g^{\prime \prime}(t) & =\frac{6}{t^{4}} \sum_{i} \frac{n_{i}^{2}}{2 r_{i}^{2}}-\frac{2}{t^{3}}\left(\sum_{i} \frac{Z}{r_{i}}-\sum_{i \neq j} \frac{1}{2 r_{i j}}\right), \\
g^{\prime \prime}\left(t^{*}\right) & =\frac{1}{t^{* 4}} \sum_{i} \frac{n_{i}^{2}}{r_{i}^{2}}>0 .
\end{aligned}
$$

Therefore $g(t)$ has only a global minimum at $t^{*}>0$, and $\phi\left(t^{*}\right)=E\left(t^{*} \boldsymbol{R}\right)$ is given as in (2.4).

Remark 1. If $\boldsymbol{R} \notin \mathcal{S}_{n} \cup \mathcal{S}_{Z}^{n}$, then for $t>0$,

$$
g(t)=E(t \boldsymbol{R})=\frac{1}{t^{2}} \sum_{i} \frac{n_{i}^{2}}{r_{i}^{2}}+\frac{1}{t}\left(-\sum_{i} \frac{Z}{r_{i}}+\sum_{i \neq j} \frac{1}{2 r_{i j}}\right) .
$$

This function $g(t)$ is always positive and monotonic for $t \in(0, \infty)$ such that $g^{\prime}(t)=0$ has no solution $t \in \mathbb{R}_{+}$. In fact, $g^{\prime}(t)<0$ on $\mathbb{R}_{+}$, i.e., $g$ is strictly decreasing. Since $g(t)$ is smooth on $\mathbb{R}_{+}$, $\lim _{t \rightarrow 0} g(t)=+\infty$ and $\lim _{t \rightarrow \infty} g(t)=0$, we have $g(t) \geq 0$ for any $t \in \mathbb{R}_{+}$.

From (2.1), by completing the square we have

$$
E(\boldsymbol{R})=\sum_{i}\left(\frac{n_{i}}{\sqrt{2} r_{i}}-\frac{\sqrt{2} Z}{n_{i}}\right)^{2}+\sum_{i \neq j} \frac{1}{2 r_{i j}}-\sum_{i} \frac{2 Z^{2}}{n_{i}^{2}} \geq-\sum_{i} \frac{2 Z^{2}}{n_{i}^{2}},
$$

therefore $E(\boldsymbol{R})$ is bounded from below, its infimum exists and we have

$$
\mu_{n}^{Z}:=\inf _{\boldsymbol{R} \in \mathbb{R}^{3 n} \backslash \mathcal{S}_{n}} E_{n}^{Z}(\boldsymbol{R})=\inf _{\boldsymbol{R} \in \mathcal{S}_{Z}^{n}} E_{n}^{Z}(\boldsymbol{R})<0 .
$$

We introduce the following gradient notation: Let $f\left(x_{1}, x_{2}, \ldots, x_{n}\right)$ be any scalar valued function of $n$ variables. For any variables $x_{i_{1}} x_{i_{2}}, \ldots, x_{i_{k}}$, where $1 \leq i_{1}<i_{2}<\cdots<i_{k} \leq n$, we denote the 
gradient of $f$ with respect to these variables as

$$
D_{\left(x_{i_{1}} x_{i_{2}}, \ldots, x_{i_{k}}\right)} f\left(x_{1}, x_{2}, \ldots, x_{n}\right)=\left[\begin{array}{l}
\frac{\partial f}{\partial x_{i_{1}}}\left(x_{1}, x_{2}, \ldots, x_{n}\right) \\
\frac{\partial f}{\partial x_{i_{2}}}\left(x_{1}, x_{2}, \ldots, x_{n}\right) \\
\vdots \\
\frac{\partial f}{\partial x_{i_{k}}}\left(x_{1}, x_{2}, \ldots, x_{n}\right)
\end{array}\right],
$$

which is regarded as a column vector. If $\left(x_{i_{1}}, x_{i_{2}}, \ldots, x_{i_{k}}\right)=\left(x_{1}, x_{2}, \ldots, x_{n}\right)$, i.e., all the variables $x_{1}, x_{2}, \ldots, x_{n}$ are included, then we simply write the above as $D f$, i.e.,

$$
D f=\left[\begin{array}{ll}
\partial f / \partial x_{1} & \partial f / \partial x_{2} \ldots \partial f / \partial x_{n}
\end{array}\right]^{T}
$$

(where $T$ means transpose). A point $\boldsymbol{y} \in \mathbb{R}^{n}$ is said to be a critical point of $f$ if $D f(y)=\mathbf{0}$. Obviously, any local or global maximum or minimum of $E$ is a critical point, but many critical points of $E$ may be of the saddle type which are physically unstable.

Later, we will also need to utilize the Hessian matrix of $f$. The Hessian matrix $H$ of $f$ is an $n \times n$ matrix whose $(i, j)$-entry, $H_{i j}$, is $H_{i j} \equiv \partial^{2} f /\left(\partial x_{i} \partial x_{j}\right)$. We denote the Hessian as $D^{2} f$, i.e.,

$$
D^{2} f=\left[\frac{\partial^{2} f}{\partial x_{i} \partial x_{j}}\right]_{1 \leq i, j \leq n}
$$

A critical point $\boldsymbol{R}^{\dagger} \in \mathbb{R}^{3 Z}$ of $E(\boldsymbol{R})$ is defined by $D E\left(\boldsymbol{R}^{\dagger}\right)=\mathbf{0}$. Thus, at a critical point we obtain the set of gradient (vector) equations

$$
D_{\boldsymbol{r}_{j}} E\left(\boldsymbol{R}^{\dagger}\right)=\mathbf{0} \text { for } \quad j=1,2, \ldots, n,
$$

i.e.,

$$
\left(\frac{n_{j}^{2}}{r_{j}^{\dagger 4}}-\frac{Z}{r_{j}^{\dagger 3}}\right) \boldsymbol{r}_{j}^{\dagger}+\sum_{\substack{k=1 \\ k \neq j}}^{n} \frac{1}{2 r_{k j}^{\dagger 3}} \boldsymbol{r}_{k j}^{\dagger}=\mathbf{0} \in \mathbf{R}^{3}, \quad j=1,2, \ldots, n .
$$

Using $\boldsymbol{r}_{k j}^{\dagger}=\boldsymbol{r}_{k}^{\dagger}-\boldsymbol{r}_{j}^{\dagger}$, we can write (2.11) alternatively as

$$
\left(\frac{n_{j}^{2}}{r_{j}^{\dagger 4}}-\frac{Z}{r_{j}^{\dagger 3}}+\sum_{\substack{k=1 \\ k \neq j}}^{n} \frac{1}{2 r_{k j}^{\dagger 3}}\right) \boldsymbol{r}_{j}^{\dagger}-\sum_{\substack{k=1 \\ k \neq j}}^{n} \frac{1}{2 r_{k j}^{\dagger 3}} \boldsymbol{r}_{k}^{\dagger}=\mathbf{0}, \quad j=1,2, \ldots, n .
$$

These constitute the equations for the steady states of electron orbits, based on the Bohr model. In particular, if $\boldsymbol{R}^{*}$ is a global minimum of $E$ established in the preceding section, then $\boldsymbol{R}^{*}$ is necessarily a critical point and so the equations in (2.11) or (2.12) hold, with the "†" signs therein replaced by "*"s.

Theorem 1 (Virial). Let $\boldsymbol{R}^{\dagger}=\left(\boldsymbol{r}_{1}^{\dagger}, \boldsymbol{r}_{2}^{\dagger}, \ldots, \boldsymbol{r}_{n}^{\dagger}\right)$ be a critical point of E satisfying $D E\left(\boldsymbol{R}^{\dagger}\right)=\mathbf{0} \in$ $\mathbb{R}^{3 n}$, then so is $\mathcal{R} \boldsymbol{R}^{\dagger}$ for any 3-D rotation $\mathcal{R}$, and $\boldsymbol{R}^{\dagger} \in \mathcal{S}_{Z}^{n}$. The ground state energy value is given by

$$
E\left(\boldsymbol{R}^{\dagger}\right)=-\sum_{i=1}^{n} \frac{n_{i}^{2}}{2 r_{i}^{\dagger 2}}<0
$$

In addition, along the ray $t \boldsymbol{R}^{\dagger}$ for $t>0, E\left(t \boldsymbol{R}^{\dagger}\right)$ attains its global minimum at $t=1$. 
Proof. Consider, for $t \neq 0$,

$$
E\left(t \boldsymbol{R}^{\dagger}\right)=\sum_{i=1}^{n}\left(\frac{n_{i}^{2}}{2 t^{2} r_{i}^{\dagger 2}}-\frac{Z}{t r_{i}^{\dagger}}\right)+\sum_{\substack{i, j=1 \\ i \neq j}}^{2} \frac{1}{2 t r_{i j}^{\dagger}} .
$$

Then, because $D E\left(\boldsymbol{R}^{\dagger}\right)=\mathbf{0}$, we have

$$
\left.\frac{d}{d t} E\left(t \boldsymbol{R}^{\dagger}\right)\right|_{t=1}=D E\left(\boldsymbol{R}^{\dagger}\right) \cdot \boldsymbol{R}^{\dagger}=0
$$

i.e.,

$$
\sum_{i}\left(-\frac{n_{i}^{2}}{r_{i}^{\dagger 2}}+\frac{Z}{r_{i}^{\dagger}}\right)-\sum_{i \neq j} \frac{1}{2 r_{i j}^{\dagger}}=0
$$

and

$$
\sum_{i} \frac{Z}{r_{i}^{\dagger}}-\sum_{i \neq j} \frac{1}{2 r_{i j}^{\dagger}}=\sum_{i} \frac{n_{i}^{2}}{r_{i}^{\dagger 2}}>0,
$$

and, hence, $\boldsymbol{R}^{\dagger} \in \mathcal{S}_{Z}^{n}$. Therefore

$$
\begin{aligned}
E\left(\boldsymbol{R}^{\dagger}\right) & =\sum_{i}\left(\frac{n_{i}^{2}}{2 r_{i}^{\dagger 2}}-\frac{Z}{r_{i}^{\dagger}}\right)+\sum_{i \neq j} \frac{1}{2 r_{i j}^{\dagger}} \\
& =\left[\sum_{i}\left(\frac{n_{i}^{2}}{r_{i}^{\dagger 2}}-\frac{Z}{r_{i}^{\dagger}}\right)+\sum_{i \neq j} \frac{1}{2 r_{i j}}\right]-\sum_{i} \frac{n_{i}^{2}}{2 r_{i}^{\dagger 2}} \\
& =-\sum_{i=1}^{Z} \frac{n_{i}^{2}}{2 r_{i}^{\dagger 2}} .
\end{aligned}
$$

But from the proof of Lemma 1, the derivative of function $g(t)=E\left(t \boldsymbol{R}^{\dagger}\right), g^{\prime}(t)$, has only one zero $t^{*}$ satisfying $g^{\prime}\left(t^{*}\right)=0$. Thus $t^{*}=1$, and $t^{*}=1$ necessarily corresponds to the global minimum of $g(t)$.

The proof is complete.

Corollary 1. Let $\boldsymbol{R}^{\dagger}=\left(\boldsymbol{r}_{1}^{\dagger}, \ldots, \boldsymbol{r}_{n}^{\dagger}\right)$ be a critical point of E satisfying Theorem 1. Define an $n \times n$ matrix $M^{\dagger}$ whose $(j, k)$-entries $M_{j k}$ are given by

$$
M_{j k}^{\dagger}= \begin{cases}-\frac{1}{2 r_{k j}^{\dagger 3},} & k \neq j ; \\ \frac{n_{k}^{2}}{r_{k}^{\dagger 4}}-\frac{Z}{r_{k}^{\dagger 3}}+\sum_{\substack{i=1 \\ i \neq k}}^{Z} \frac{1}{2 r_{k i}^{\dagger 3}}, & j=k\end{cases}
$$

Then $\operatorname{det} M^{\dagger}=0$.

Proof. Note that these $M_{j k}^{\dagger}$ 's are the coefficients appearing in (2.12). As the matrix equation $M^{\dagger} \boldsymbol{\alpha}=\mathbf{0} \in \mathbb{R}^{n}$ has at least a nontrivial solution $\boldsymbol{\alpha} \in \mathbb{R}^{n}, \boldsymbol{\alpha} \neq \mathbf{0}$, where $\boldsymbol{\alpha}$ is a vector formed by all of the $i^{\text {th }}$ components of the vectors in $\left(\boldsymbol{r}_{1}^{\dagger}, \boldsymbol{r}_{2}^{\dagger}, \ldots, \boldsymbol{r}_{n}^{\dagger}\right)$, with $i=1$, or 2 , or 3 , we must have the determinant of $M^{\dagger}$ equal to 0. 
From (2.14), we obtain that the set

$$
\mathcal{N}_{Z}^{n}=\left\{\boldsymbol{R}=\left(\boldsymbol{r}_{1}, \ldots, \boldsymbol{r}_{n}\right) \in \mathbb{R}^{3 n} \backslash \mathcal{S}_{n} \mid \sum_{k=1}^{n}\left[\frac{Z}{r_{k}}-\frac{n_{i}^{2}}{r_{k}^{2}}\right]=\sum_{\substack{j, k=1 \\ j \neq k}}^{n} \frac{1}{2 r_{j k}}\right\},
$$

contains all critical points of $E_{n}^{Z} \cdot \mathcal{N}_{Z}^{n}$ is a closed nonempty subset of $\mathbb{R}^{3 n}$, and $\mathcal{N}_{Z}^{n} \subset \mathcal{S}_{Z}^{n}$. We then have

$$
\inf _{\boldsymbol{R} \in \mathcal{S}_{Z}^{n}} E_{n}^{Z}(\boldsymbol{R})=\inf _{\boldsymbol{R} \in \mathcal{N}_{Z}^{n}} E_{n}^{Z}(\boldsymbol{R}) .
$$

Furthermore, we obtain from $(2.4)$

$$
E_{n}^{Z}(\boldsymbol{R})=-\frac{1}{2} \sum_{i=1}^{n} \frac{n_{i}^{2}}{r_{i}^{2}}<0, \quad \forall \boldsymbol{R}=\left(\boldsymbol{r}_{1}, \ldots, \boldsymbol{r}_{n}\right) \in \mathcal{N}_{Z}^{n}
$$

When $n=1$, define $\mathcal{N}_{Z}^{1}=\left\{\boldsymbol{r}_{1} \in \mathbb{R}^{3} \mid r_{1}=n_{1}^{2} / Z\right\}$. For any $\boldsymbol{r} \in \mathcal{N}_{Z}^{1}$,

$$
E_{1}^{Z}(\boldsymbol{r})=-\frac{Z^{2}}{2 n_{1}^{2}}=\inf _{\boldsymbol{r}_{1} \in \mathbb{R}^{3} \backslash \mathcal{S}_{1}} E_{1}^{Z}\left(\boldsymbol{r}_{1}\right) .
$$

Lemma 2. Let $n \geq 2$ and $Z=n$, then

$$
\mu_{n}^{Z} \leq \mu_{k}^{Z}, \quad \forall k: 1 \leq k \leq n-1 .
$$

If $\mu_{k}^{Z}=E_{k}^{Z}\left(\boldsymbol{r}_{1}^{*}, \ldots, \boldsymbol{r}_{k}^{*}\right)$ for some $\left(\boldsymbol{r}_{1}^{*}, \ldots, \boldsymbol{r}_{k}^{*}\right) \in\left(\mathbb{R}^{3}\right)^{k}$, then

$$
\mu_{n}^{Z}<\mu_{k}^{Z}, \quad \forall 1 \leq k \leq n-1 .
$$

Proof. For any $\left(\boldsymbol{r}_{1}, \ldots, \boldsymbol{r}_{k}\right) \in \mathcal{S}_{Z}^{k}$ and $\left(\boldsymbol{r}_{k+1}, \ldots, \boldsymbol{r}_{n}\right) \in \mathcal{S}_{Z-k}^{n-k}$, we have

$$
\begin{aligned}
& E_{n}^{Z}\left(\boldsymbol{r}_{1}, \ldots, \boldsymbol{r}_{k}, t \boldsymbol{r}_{k+1}, \ldots, t \boldsymbol{r}_{n}\right) \\
= & \left\{\sum_{j=1}^{k}\left[\frac{n_{j}^{2}}{2 r_{j}^{2}}-\frac{Z}{r_{j}}\right]+\sum_{\substack{j, i=1 \\
j \neq i}}^{k} \frac{1}{2 r_{i j}}\right\} \\
& +\left\{\sum_{j=k+1}^{n}\left[\frac{n_{j}^{2}}{2 t^{2} r_{j}^{2}}-\frac{Z}{t r_{j}}\right]+\sum_{\substack{j, i=k+1 \\
j \neq i}}^{n} \frac{1}{2 t r_{i j}}+\sum_{j=k+1}^{n} \sum_{i=1}^{k} \frac{1}{\left|t \boldsymbol{r}_{j}-\boldsymbol{r}_{i}\right|}\right\} \\
\equiv & \Delta_{1}+\frac{1}{t} \Delta_{2}
\end{aligned}
$$

Note that

$$
\begin{aligned}
\lim _{t \rightarrow \infty} \Delta_{2} & =\lim _{t \rightarrow \infty}\left\{\sum_{j=k+1}^{n}\left[\frac{n_{j}^{2}}{2 t r_{j}^{2}}-\frac{Z}{r_{j}}\right]+\sum_{\substack{j, i=k+1 \\
j \neq i}}^{n} \frac{1}{2 r_{i j}}+\sum_{j=k+1}^{n} \sum_{i=1}^{k} \frac{t}{\left|\boldsymbol{r}_{j}-\boldsymbol{r}_{i}\right|}\right\} \\
& =-\left(\sum_{j=k+1}^{n} \frac{Z-k}{r_{j}}-\sum_{\substack{j, i=k+1 \\
j \neq i}}^{n} \frac{1}{2 r_{i j}}\right) .
\end{aligned}
$$


In the special case when $k=n-1$, we have $\boldsymbol{r}_{n} \in \mathcal{S}_{Z-n+1}^{1}$ and then

$$
\lim _{t \rightarrow \infty} \Delta_{2}=-\frac{Z-n+1}{r_{n}}<0
$$

but if $1 \leq k \leq n-2$, which occurs only when $n \geq 3$, then $\left(\boldsymbol{r}_{k+1}, \ldots, \boldsymbol{r}_{n}\right) \in \mathcal{S}_{Z-k}^{n-k}$ implies

$$
\lim _{t \rightarrow \infty} \Delta_{2}=-\left(\sum_{j=k+1}^{n} \frac{Z-k}{r_{j}}-\sum_{\substack{j, i=k+1 \\ j \neq i}}^{n} \frac{1}{2 r_{i j}}\right)<0 .
$$

Thus we obtain, for any $\left(\boldsymbol{r}_{k+1}, \ldots, \boldsymbol{r}_{n}\right) \in \mathcal{S}_{Z-k}^{n-k}$

$$
\frac{1}{t} \Delta_{2}<0, \quad \text { for } t \text { sufficiently large. }
$$

Hence, we have for any $\left(\boldsymbol{r}_{1}, \ldots, \boldsymbol{r}_{k}\right) \in \mathcal{S}_{Z}^{k}$, for $t$ sufficiently large,

$$
\mu_{n}^{Z} \leq E_{n}^{Z}\left(\boldsymbol{r}_{1}, \ldots, \boldsymbol{r}_{k}, \boldsymbol{t r}_{k+1}, \ldots, t \boldsymbol{r}_{n}\right)<\left(\sum_{j=1}^{k}\left[\frac{n_{j}^{2}}{2 r_{j}^{2}}-\frac{Z}{r_{j}}\right]+\sum_{\substack{j, i=1 \\ j \neq i}}^{k} \frac{1}{2 r_{i j}}\right) .
$$

Thus

$$
\mu_{n}^{Z} \leq \mu_{k}^{Z}
$$

If $\mu_{k}^{Z}=E_{k}^{Z}\left(\boldsymbol{r}_{1}^{*}, \ldots, \boldsymbol{r}_{k}^{*}\right)$ for some $\left(\boldsymbol{r}_{1}^{*}, \ldots, \boldsymbol{r}_{k}^{*}\right) \in\left(\mathbb{R}^{3}\right)^{k}$, then (2.19) and (2.20) leads to

$$
\mu_{n}^{Z}<\mu_{k}^{Z}
$$

Theorem 2. Let $Z=n$. There exists an $\boldsymbol{R}^{*}=\left(\boldsymbol{r}_{1}^{*}, \ldots, \boldsymbol{r}_{n}^{*}\right) \in \mathbb{R}^{3 n}$ such that

$$
E_{n}^{Z}\left(\boldsymbol{R}^{*}\right)=\inf _{\boldsymbol{R} \in \mathbb{R}^{3 n} \backslash \mathcal{S}_{n}} E_{n}^{Z}(\boldsymbol{R}) .
$$

Proof. Since the case of $n=1$ is trivial, we assume $n \geq 2$. From (2.8) and (2.17), we only need to prove that there exists an $\boldsymbol{R}^{*}=\left(r_{1}^{*}, \ldots, r_{n}^{*}\right) \in \mathcal{N}_{Z}^{n}$ such that

$$
E_{n}^{Z}\left(\boldsymbol{R}^{*}\right)=\inf _{\boldsymbol{R} \in \mathcal{N}_{Z}^{n}} E_{n}^{Z}(\boldsymbol{R})=\mu_{n}^{Z}
$$

Let $\left\{\boldsymbol{R}_{m}\right\} \subset \mathcal{N}_{Z}^{n}$ be a minimizing sequence such that $E_{n}^{Z}\left(\boldsymbol{R}_{m}\right) \rightarrow \mu_{n}^{Z}$ as $m \rightarrow \infty$. We first show that $\left\{\boldsymbol{R}_{m}\right\}$ is bounded.

Suppose $\left\{\boldsymbol{R}_{m}\right\}$ is unbounded. Let $\boldsymbol{R}_{m}=\left(\boldsymbol{r}_{1}^{m}, \ldots, \boldsymbol{r}_{n}^{m}\right)$. By replacing $\left\{\boldsymbol{R}_{m}\right\}$ with its subsequence, if necessary, essentially two cases need to be considered:

(a) $\left|\boldsymbol{r}_{k}^{m}\right| \rightarrow+\infty, 1 \leq k \leq n$; and

(b) there exist $M>0$ and $k_{0}: 1 \leq k_{0} \leq n-1$, such that

$$
\begin{cases}r_{k}^{m} \rightarrow+\infty, & 1 \leq k \leq k_{0} \\ r_{k}^{m} \leq M, & k_{0}+1 \leq k \leq n\end{cases}
$$


In general, the index set $\{1,2, \ldots, n\}$ has two disjoint subsets such that $r_{k}^{m} \rightarrow \infty$ and $r_{k}^{m}<M$, respectively, as $m \rightarrow \infty$ for $k$ in each of these two index subsets. But the proof is the same.

For case (a), since $\left\{\mathbf{R}_{m}\right\} \subset \mathcal{N}_{Z}^{n}$ and (2.18), we have

$$
\mu_{n}^{Z}=\lim _{m \rightarrow \infty} E_{n}^{Z}\left(\boldsymbol{R}_{m}\right)=\lim _{m \rightarrow \infty}\left\{-\frac{1}{2} \sum_{i=1}^{n} \frac{n_{i}^{2}}{r_{i}^{m}}\right\}=0 .
$$

This contradicts $\mu_{n}^{Z}<0$ from (2.8). Thus, case (a) is impossible.

For case (b), we can further assume that $\boldsymbol{r}_{k}^{m} \rightarrow \boldsymbol{r}_{k}^{*}$ for $k_{0}+1 \leq k \leq n$. Then we have

$$
\begin{aligned}
\mu_{n}^{Z} & =\lim _{m \rightarrow \infty} E_{n}^{n}\left(\boldsymbol{R}_{m}\right) \\
& =\lim _{m \rightarrow \infty}\left\{\sum_{i=1}^{n}\left(\frac{n_{i}^{2}}{2\left(r_{i}^{m}\right)^{2}}-\frac{Z}{r_{i}^{m}}\right)+\sum_{\substack{i, j=1 \\
i \neq j}}^{n} \frac{1}{2 r_{i j}^{m}}\right\} \\
& =\left\{\sum_{k=k_{0}+1}^{n}\left[\frac{n_{k}^{2}}{2\left(r_{k}^{*}\right)^{2}}-\frac{Z}{r_{k}^{*}}\right]+\sum_{\substack{j, k=k_{0}+1 \\
j \neq k}}^{n} \frac{1}{2 r_{j k}^{*}}\right\}+\lim _{m \rightarrow \infty} \sum_{\substack{j, k=1 \\
j \neq k}}^{k_{0}} \frac{1}{2 r_{j k}^{m}} \\
& \geq \mu_{n-k_{o}}^{Z}+\lim _{m \rightarrow \infty} \sum_{\substack{j, k=1 \\
j \neq k}}^{k_{0}} \frac{1}{2 r_{j k}^{m}} \geq \mu_{n-k_{0}}^{Z},
\end{aligned}
$$

By applying the first part of Lemma 2, we must have

$$
\lim _{m \rightarrow \infty} \sum_{\substack{j, k=1 \\ j \neq k}}^{k_{0}} \frac{1}{r_{j k}^{m}}=0
$$

and

$$
\mu_{n}^{Z}=\mu_{n-k_{0}}^{Z}=\sum_{k=k_{0}+1}^{n}\left[\frac{n_{i}^{2}}{2\left(r_{k}^{*}\right)^{2}}-\frac{Z}{r_{k}^{*}}\right]+\sum_{\substack{j, k=k_{0}+1 \\ j \neq k}}^{n} \frac{1}{2 r_{j k}^{*}},
$$

which contradicts the second part of Lemma 2. Thus, case (b) is also impossible.

Therefore $\left\{\boldsymbol{R}_{m}\right\}$ is bounded and contains a convergent subsequence, denoted by $\left\{\boldsymbol{R}_{m}\right\}$ again, such that $\boldsymbol{R}^{m} \rightarrow \boldsymbol{R}^{*} \in \mathbb{R}^{3 n}$. Since $\mathcal{N}_{Z}^{n}$ is a closed subset of $\mathbb{R}^{3 n}$, we have $\boldsymbol{R}^{*} \in \mathcal{N}_{Z}^{n}$, and

$$
E_{n}^{n}\left(\boldsymbol{R}^{*}\right)=\mu_{n}^{Z}=\inf _{\boldsymbol{R} \in \mathbb{R}^{3 n} \backslash \mathcal{S}_{n}} E_{n}^{Z}(\boldsymbol{R})
$$

Corollary 2. Let $Z=n$. For any nontrivial subspace $V$ of $\mathbb{R}^{3}$, the energy function $E_{n}^{Z}$ defined in (2.1) attains a minimum in $V^{n}$. 


\section{Critical Points of the Energy E: Stable and Unstable Electron Configurations}

From here throughout the rest of the paper for all practical interest we assume $n=Z$ for $E_{n}^{Z}$, which will be written simply as $E$.

In order to distinguish any global (or, possibly local) minimizer $R^{*}$ from a saddle-type critical point $\boldsymbol{R}^{\wedge}$ of $E$, we need to examine whether the $(3 Z) \times(3 Z)$ Hessian matrix $D^{2} E$ (cf. (2.9)) is (semi-) positive-definite at $\boldsymbol{R}^{*}$, as the second order Taylor approximation gives

$$
\begin{aligned}
E(\boldsymbol{R})= & E\left(\boldsymbol{R}^{*}\right)+D E\left(\boldsymbol{R}^{*}\right) \cdot\left(\boldsymbol{R}-\boldsymbol{R}^{*}\right)+\frac{1}{2}\left(\boldsymbol{R}-\boldsymbol{R}^{*}\right)^{T} \cdot D^{2} E\left(\boldsymbol{R}^{*}\right) \cdot\left(\boldsymbol{R}-\boldsymbol{R}^{*}\right) \\
& +\mathcal{O}\left(\left|\boldsymbol{R}-\boldsymbol{R}^{*}\right|^{3}\right) \\
= & E\left(\boldsymbol{R}^{*}\right)+\frac{1}{2}\left(\boldsymbol{R}-\boldsymbol{R}^{*}\right)^{T} \cdot D^{2} E\left(\boldsymbol{R}^{*}\right) \cdot\left(\boldsymbol{R}-\boldsymbol{R}^{*}\right)+\mathcal{O}\left(\left|\boldsymbol{R}-\boldsymbol{R}^{*}\right|^{3}\right) \\
& \text { for }\left|\boldsymbol{R}-\boldsymbol{R}^{*}\right| \text { small, }
\end{aligned}
$$

requiring that

$$
\begin{aligned}
& \boldsymbol{R}^{T} \cdot D^{2} E\left(\boldsymbol{R}^{*}\right) \cdot \boldsymbol{R} \geq 0 \text { for any } \quad \boldsymbol{R} \in \mathbb{R}^{3 Z}, \\
& \text { for } \boldsymbol{R}^{*} \text { to be a local or global minimum. }
\end{aligned}
$$

Following the terminology of calculus of variations, we say that a critical point $\boldsymbol{R}^{\wedge}$ is nondegenerate if $D_{\boldsymbol{R}}^{2} E\left(\boldsymbol{R}^{\wedge}\right)$ is an invertible (i.e., nonsingular) $(3 Z) \times(3 Z)$ matrix. Otherwise, $\boldsymbol{R}^{\wedge}$ is said to be a degenerate critical point. Thus, a critical point is degenerate if and only if $D_{\boldsymbol{R}}^{2} E\left(\boldsymbol{R}^{\wedge}\right)$ has 0 as its eigenvalue. At a critical point $\boldsymbol{R}^{\wedge}$, the number of negative eigenvalues is called the Morse index of that critical point. If a critical point $\boldsymbol{R}^{\wedge}$ is non-degenerate and has a Morse index greater than or equal to 1 , then $\boldsymbol{R}^{\wedge}$ must correspond to a saddle point of $E(\boldsymbol{R})$. But here all the critical points are degenerate according to the following.

Theorem 3 (Degeneracy of critical points). Any critical point $\boldsymbol{R}^{\wedge}$ of $E(\boldsymbol{R})$ is degenerate. The dimension of degeneracy is at least 2.

Proof. Any critical point $\boldsymbol{R}_{0}^{\wedge}$ satisfies (2.10) and, according to Theorem $1, \mathcal{R} \boldsymbol{R}^{\wedge}$ is also a critical point for any $3-\mathrm{D}$ rotation $\mathcal{R}$ :

$$
D E\left(\mathcal{R} \boldsymbol{R}_{0}^{\wedge}\right)=\mathbf{0} \in \mathbb{R}^{3 Z} .
$$

From the theory of Lie groups in $\mathbb{R}^{3}$, we know that the rotation group $S O(3)$ (i.e., the special orthogonal group) in $\mathbb{R}^{3}$ can be parametrized by two independent parameters $\left(\theta_{1}, \theta_{2}\right)$. So we may write $\mathcal{R}=\mathcal{R}\left(\theta_{1}, \theta_{2}\right)$ and define

$$
\boldsymbol{R}^{\wedge}\left(\theta_{1}, \theta_{2}\right) \equiv \mathcal{R}\left(\theta_{1}, \theta_{2}\right) \boldsymbol{R}_{0}^{\wedge}
$$

Then by (3.1),

$$
D E\left(\boldsymbol{R}^{\wedge}\left(\theta_{1}, \theta_{2}\right)\right)=\mathbf{0} \text { for all } \theta_{1} \text { and } \theta_{2} .
$$

Thus, by the chain rule,

$$
\frac{\partial}{\partial \theta_{j}} D E\left(\boldsymbol{R}^{\wedge}\left(\theta_{1}, \theta_{2}\right)\right)=D^{2} E\left(\boldsymbol{R}^{\wedge}\left(\theta_{1}, \theta_{2}\right)\right) \cdot\left[\frac{\partial}{\partial \theta_{j}} \boldsymbol{R}^{\wedge}\left(\theta_{1}, \theta_{2}\right)\right]=\mathbf{0}, \quad j=1,2 .
$$

Since

$$
\frac{\partial}{\partial \theta_{j}} \boldsymbol{R}^{\wedge}\left(\theta_{1}, \theta_{2}\right) \neq \mathbf{0} \quad \text { for } \quad j=1,2
$$


and $\frac{\partial}{\partial \theta_{1}} \boldsymbol{R}^{\wedge}\left(\theta_{1}, \theta_{2}\right)$ and $\frac{\partial}{\partial \theta_{2}} \boldsymbol{R}^{\wedge}\left(\theta_{1}, \theta_{2}\right)$ are linearly independent, because by holding $\theta_{1}=\theta_{1}^{0}$ and $\theta_{2}=\theta_{2}^{0}, \boldsymbol{R}^{\wedge}\left(\theta_{1}, \theta_{2}^{0}\right)$ and $\boldsymbol{R}^{\wedge}\left(\theta_{1}^{0}, \theta_{2}\right)$ form two independent trajectories when only one of $\theta_{1}$ and $\theta_{2}$ is allowed to vary, and $\frac{\partial}{\partial \theta_{1}} \boldsymbol{R}^{\wedge}\left(\theta_{1}, \theta_{2}^{0}\right)$ and $\frac{\partial}{\partial \theta_{2}} \boldsymbol{R}^{\wedge}\left(\theta_{1}^{0}, \theta_{2}\right)$ are the tangent vectors along these two independent trajectories. We conclude from (3.4) that the Hessian matrix $D^{2} E\left(\boldsymbol{R}^{\wedge}\right)$ has two linearly independent eigenvectors corresponding to the eigenvalue 0 . The proof is complete.

The determination of all critical point $\boldsymbol{R}^{\dagger}$ from either (2.11) or analytically is no easy task. The following two theorems provide a systematic way to construct (unstable) saddle-type critical points which are not global minima.

Define a subset $\mathbb{R}_{x}^{3 Z}$ of $\mathbb{R}^{3 Z}$ by

$$
\begin{aligned}
\mathbb{R}_{x}^{3 Z} & =\left\{\boldsymbol{R}=\left(\boldsymbol{r}_{1}, \boldsymbol{r}_{2}, \ldots, \boldsymbol{r}_{Z}\right) \in \mathbb{R}^{3 Z} \mid \boldsymbol{r}_{j}=\left(x_{j}, y_{j}, z_{j}\right)^{T}=\left(x_{j}, 0,0\right), \text { for } j=1,2, \ldots, Z\right. \\
& \left.x_{j} \in \mathbb{R}\right\} .
\end{aligned}
$$

So $\mathbb{R}_{x}^{3 Z}$ is a $Z$-dimensional subspace of $\mathbb{R}^{3 D}$. The subspaces $\mathbb{R}_{y}^{3 Z}$ and $\mathbb{R}_{z}^{3 Z}$ can be defined likewise.

Theorem 4. The minimization problem

$$
\min _{\boldsymbol{R} \in \mathbb{R}_{x}^{3 Z}} E(\boldsymbol{R})
$$

has at least a (global) minimizer $\boldsymbol{R}_{x}^{*} \in \mathbb{R}_{x}^{3 Z}$. This $\boldsymbol{R}_{x}^{*}$ is also a critical point of $E$ in $\mathbb{R}^{3 Z}$, i.e., $D E\left(\boldsymbol{R}_{x}^{*}\right)=\mathbf{0}$. In fact, any critical point $\boldsymbol{R}_{x}^{\dagger}$ of $E(\boldsymbol{R})$ in $\mathbb{R}_{x}^{3 Z}$ is also a critical point of $E$ in $\mathbb{R}^{3 Z}$, i.e., $D E\left(\boldsymbol{R}_{x}^{\dagger}\right)=\mathbf{0}$. The same is true if $\mathbb{R}_{x}^{3 Z}$ is replaced by $\mathbb{R}_{y}^{3 Z}$ or $\mathbb{R}_{z}^{3 Z}$.

Proof. Because $\mathbb{R}_{x}^{3 Z}$ is a closed subspace of $\mathbb{R}^{3 Z}$, we can establish that (3.7) has a minimizer $\boldsymbol{R}_{x}^{*}$ in $\mathbb{R}_{x}^{3 Z}$ by Corollary 2. The same conclusion follows for $\mathbb{R}_{y}^{3 Z}$ and $\mathbb{R}_{y}^{3 Z}$.

We now show how $\boldsymbol{R}_{x}^{*}$ satisfies $D E\left(\boldsymbol{R}_{x}^{*}\right)=\mathbf{0}$. Since $\boldsymbol{R}_{x}^{*}$ solves (3.7), we have the gradient equations

$$
\left.\frac{\partial}{\partial x_{j}} E(\boldsymbol{R})\right|_{\boldsymbol{R}=\boldsymbol{R}_{x}^{*}}=0, \text { for } j=1,2, \ldots, Z \text {; cf. (3.6) for } x_{j} .
$$

The above gives

$$
\left(\frac{n_{j}^{2}}{x_{j}^{* 4}}-\frac{Z}{x_{j}^{* 3}}+\sum_{\substack{k=1 \\ k \neq j}}^{Z} \frac{1}{2\left|x_{k}^{*}-x_{j}^{*}\right|^{3}}\right) x_{j}^{*}-\sum \frac{1}{2\left|x_{k}^{*}-x_{j}^{*}\right|^{3}} x_{k}^{*}=0, \quad j=1,2, \ldots, Z,
$$

where $x_{j}^{*}$ 's are the first components of $\boldsymbol{r}_{j}^{*}$, with $\boldsymbol{R}_{x}^{*}=\left(\boldsymbol{r}_{1}^{*}, \boldsymbol{r}_{2}^{*}, \ldots, \boldsymbol{r}_{Z}^{*}\right)$.

Note that (3.8) just represents the first component (i.e., related to $x$ ) of the vector equations (2.12), with "†" therein replaced by " $*$ ". But the second and third components (related to $y$ and $z$ ) are automatically satisfied because $y_{j}=z_{j}=0$ for $j=1,2, \ldots, Z$.

Next, define the following subsets of $\mathbb{R}^{3 Z}$ :

$$
\begin{gathered}
\mathbb{R}_{x, y}^{3 Z}=\left\{\boldsymbol{R}=\left(\boldsymbol{r}_{1}, \boldsymbol{r}_{2}, \ldots, \boldsymbol{r}_{Z}\right) \in \mathbb{R}^{3 Z} \mid \boldsymbol{r}_{j}=\left(x_{j}, y_{j}, z_{j}\right)=\left(x_{j}, y_{j}, 0\right), \text { for } j=1,2, \ldots, Z ;\right. \\
\left.x_{j}, y_{j} \in \mathbb{R}\right\}, \\
\mathbb{R}_{x, z}^{3 Z}=\left\{\boldsymbol{R}=\left(\boldsymbol{r}_{1}, \boldsymbol{r}_{2}, \ldots, \boldsymbol{r}_{z}\right) \in \mathbb{R}^{3 Z} \mid \begin{array}{r}
\boldsymbol{r}_{j}= \\
\left(x_{j}, y_{j}, z_{j}\right)=\left(x_{j}, 0, z_{j}\right), \text { for } j=1,2, \ldots, Z ; \\
\left.x_{j}, z_{j} \in \mathbb{R}\right\},
\end{array}\right. \\
\mathbb{R}_{y, z}^{3 Z}=\left\{\boldsymbol{R}=\left(\boldsymbol{r}_{1}, \boldsymbol{r}_{2}, \ldots, \boldsymbol{r}_{Z}\right) \in \mathbb{R}^{3 Z} \mid \begin{array}{c}
\boldsymbol{r}_{j}= \\
\left(x_{j}, y_{j}, z_{j}\right)=\left(0, y_{j}, z_{j}\right), \text { for } j=1,2, \ldots, Z ; \\
\left.y_{j}, z_{j} \in \mathbb{R}\right\} .
\end{array}\right.
\end{gathered}
$$


Theorem 5. The minimization problem

$$
\min _{\boldsymbol{R} \in \mathbb{R}_{x, y}^{3 Z}} E(\boldsymbol{R})
$$

has at least a (global) minimizer $\boldsymbol{R}_{x, y}^{*} \in \mathbb{R}_{x, y}^{3 Z}$. This $\boldsymbol{R}_{x, y}^{*}$ is a critical point of $E(\boldsymbol{R})$ in $\mathbb{R}^{3 Z}$, i.e., $D E\left(\boldsymbol{R}_{x, y}^{*}\right)=0$. In fact, any critical point $\boldsymbol{R}_{x, y}^{\dagger}$ of $E(\boldsymbol{R})$ in $\mathbb{R}_{x, y}^{3 Z}$ is also a critical point of $E(\boldsymbol{R})$ in $\mathbb{R}^{3 Z}$, i.e., $D E\left(\boldsymbol{R}_{x, y}^{\dagger}\right)=\mathbf{0}$. The same theorem holds if we replace $\mathbb{R}_{x, y}^{3 D}$ above by $\mathbb{R}_{x, z}^{3 D}$ or $\mathbb{R}_{y, z}^{3 D}$.

Proof. Same as that for Theorem 4.

Corollary 3. Let $V$ be a (coordinate) subspace of $\mathbb{R}^{3}$ with dimension 1 or 2. Then any critical point of $E$ on $V^{Z}$ is a critical point of $E$ on $\mathbb{R}^{3 Z}$.

Proof. Any such $V$ can be obtained by a rotation from $\mathbb{R}_{x}^{3 Z}$ or $\mathbb{R}_{x, y}^{3 Z}$, respectively, if $V$ has dimension of, respectively, 1 and 2 . The corollary follows because $E$ is rotationally invariant.

With the aid of Theorems 4 and 5, we will be able to obtain many unstable critical points of $E(\boldsymbol{R})$ in $\mathbb{R}^{3 Z}$. See Examples 2 and 3 in Section 5.

We conjecture that the energy function $E$ as defined in (1.5)-(1.10) has only finitely many critical points $\boldsymbol{R}^{\dagger}$ satisfying $D E\left(\boldsymbol{R}^{\dagger}\right)=\mathbf{0}$, which are not rotationally equivalent. But we don't yet have a proof.

\section{Coplanarity of Stable or Unstable Electron Configurations}

Numerical results (see Example in Section 5) indicate that for $Z=3$ and 4, the stable electron configuration (corresponding to a global minimizer of $E$ ) has all electrons coplanar with the origin. For $Z=1$ and 2, the coplanarity is trivial. For $Z \geq 5$, numerical evidence suggests that coplanarity no longer holds.

Theorem 6 (Co-planarity of the electrons with the origin: $Z=3$ ). Let $Z=3$. If $\boldsymbol{R}^{\dagger}=$ $\left(\boldsymbol{r}_{1}^{\dagger}, \boldsymbol{r}_{2}^{\dagger}, \boldsymbol{r}_{3}^{\dagger}\right)$ is a critical point of $E(\boldsymbol{R})$ (including the global minimizer $\boldsymbol{R}^{*}$ ), then $\boldsymbol{r}_{1}^{\dagger}, \boldsymbol{r}_{2}^{\dagger}, \boldsymbol{r}_{3}^{\dagger}$, and $\mathbf{0}$ are coplanar in $\mathbb{R}^{3}$.

Proof. For clarity, we write out the system of equations (2.12):

$$
\left\{\begin{array}{l}
\left.\frac{1}{r_{1}^{\dagger 4}}-\frac{3}{r_{1}^{\dagger 3}}+\frac{1}{r_{12}^{\dagger 3}}+\frac{1}{r_{13}^{\dagger 3}}\right) \boldsymbol{r}_{1}^{\dagger}-\frac{1}{r_{12}^{\dagger 3}} \boldsymbol{r}_{2}^{\dagger}-\frac{1}{r_{13}^{\dagger 3}} \boldsymbol{r}_{3}^{\dagger}=\mathbf{0} \\
\left.\frac{1}{r_{2}^{\dagger 4}}-\frac{3}{r_{2}^{\dagger 3}}+\frac{1}{r_{12}^{\dagger 3}}+\frac{1}{r_{23}^{\dagger 3}}\right) \boldsymbol{r}_{2}^{\dagger}-\frac{1}{r_{12}^{\dagger 3}} \boldsymbol{r}_{1}-\frac{1}{r_{23}^{\dagger 3}} \boldsymbol{r}_{3}^{\dagger}=\mathbf{0} \\
\left.\frac{4}{r_{3}^{\dagger 4}}-\frac{3}{r_{3}^{\dagger 3}}+\frac{1}{r_{13}^{\dagger 3}}+\frac{1}{r_{23}^{\dagger 3}}\right) \boldsymbol{r}_{3}^{\dagger}-\frac{1}{r_{13}^{3}} \boldsymbol{r}_{1}^{\dagger}-\frac{1}{r_{23}^{\dagger 3}} \boldsymbol{r}_{2}^{\dagger}=\mathbf{0}
\end{array}\right\}
$$

If any single equation in (4.1) has all 3 coefficients of $\boldsymbol{r}_{1}^{\dagger}, \boldsymbol{r}_{2}^{\dagger}$ and $\boldsymbol{r}_{3}^{\dagger}$ to be nonzero, then any one vector in $\left\{\boldsymbol{r}_{1}^{\dagger}, \boldsymbol{r}_{2}^{\dagger}, \boldsymbol{r}_{3}^{\dagger}\right\}$ can be expressed as a linear combination of the other two vectors, and thus the proof follows. Therefore, the only possibility that $\boldsymbol{r}_{1}^{\dagger}, \boldsymbol{r}_{2}^{\dagger}, \boldsymbol{r}_{3}^{\dagger}$ and $\mathbf{0}$ are not coplanar is when

$$
\frac{n_{i}^{2}}{r_{i}^{\dagger 4}}-\frac{3}{r_{i}^{\dagger 3}}-\sum_{\substack{j \neq i \\ j=1}}^{3} \frac{1}{r_{i j}^{\dagger}}=0, \quad \text { for } \quad i=1,2,3
$$


But the above implies, from (4.2), that

$$
\left.\begin{array}{l}
\frac{1}{r_{12}^{\dagger 3}} \boldsymbol{r}_{2}^{\dagger}+\frac{1}{r_{13}^{\dagger 3}} \boldsymbol{r}_{3}^{\dagger}=\mathbf{0}, \\
\frac{1}{r_{12}^{\dagger 3}} \boldsymbol{r}_{1}^{\dagger}+\frac{1}{r_{23}^{\dagger 3}} \boldsymbol{r}_{3}^{\dagger}=\mathbf{0} \\
\frac{1}{r_{12}^{\dagger 3}} \boldsymbol{r}_{1}^{\dagger}+\frac{1}{r_{23}^{\dagger 3}} \boldsymbol{r}_{2}^{\dagger}=\mathbf{0}
\end{array}\right\}
$$

Adding up the three equations in (4.2), we have

$$
\sum_{i=}^{3}\left(\sum_{\substack{j=1 \\ j \neq i}}^{3} \frac{1}{r_{i j}^{\dagger 3}}\right) \boldsymbol{r}_{i}^{\dagger}=\mathbf{0} .
$$

Once again, any $\boldsymbol{r}_{i}^{\dagger}$ can be expressed as a linear combination of $\boldsymbol{r}_{j}^{\dagger}$ for $j \neq i$. Therefore, $\boldsymbol{r}_{1}^{\dagger}, \boldsymbol{r}_{2}^{\dagger}, \boldsymbol{r}_{3}^{\dagger}$ and $\mathbf{0}$ are coplanar.

At this time, we are not yet able to prove the coplanarity property for the case $Z=4$, which as Fig. 3 in the next section indicates, is true from numerical computation.

\section{$5 \quad$ Numerical Examples and Data}

We first list in Table 2 the comparison between the experimental values of atoms' ground-state energies and those of the Bohr energies, for atom numbers between 2 and 30. There is a reasonable agreement between these values when $Z$ is small. When the value of $Z$ increases, the deviations also grow. Nevertheless, the trend of Bohr's atom energies look good.

\begin{tabular}{|r|r|r|}
\hline \multicolumn{1}{|c|}{$\mathrm{Z}$} & Experimental & \multicolumn{1}{c|}{ Bohr's } \\
\hline 2.0 & -2.903 & -3.0615 \\
\hline 3.0 & -7.478 & -7.6889 \\
\hline 4.0 & -14.667 & -14.8377 \\
\hline 5.0 & -24.652 & -24.7906 \\
\hline 6.0 & -37.842 & -37.8128 \\
\hline 7.0 & -54.584 & -54.1540 \\
\hline 8.0 & -75.059 & -74.1726 \\
\hline 9.0 & -99.719 & -97.9746 \\
\hline 10.0 & -128.919 & -125.5152 \\
\hline 11.0 & -162.233 & -156.9173 \\
\hline 12.0 & -200.026 & -192.3112 \\
\hline 13.0 & -242.315 & -231.7757 \\
\hline 14.0 & -289.322 & -275.4952 \\
\hline 15.0 & -341.208 & -323.5122 \\
\hline 16.0 & -398.601 & -376.0176 \\
\hline 17.0 & -460.102 & -433.0846 \\
\hline 18.0 & -527.494 & -494.9136 \\
\hline 19.0 & -599.924 & -561.4195 \\
\hline
\end{tabular}




\begin{tabular}{|l|r|r|}
\hline 20.0 & -677.558 & -632.8097 \\
\hline 21.0 & -760.575 & -709.3828 \\
\hline 22.0 & -849.285 & -791.0756 \\
\hline 23.0 & -943.804 & -878.0731 \\
\hline 24.0 & -1044.315 & -970.5907 \\
\hline 25.0 & -1150.866 & -1068.6207 \\
\hline 26.0 & -1263.483 & -1172.1997 \\
\hline 27.0 & -1382.494 & -1281.5093 \\
\hline 28.0 & -1507.990 & -1396.5737 \\
\hline 29.0 & -1640.123 & -1517.7809 \\
\hline 30.0 & -1779.048 & -1644.9630 \\
\hline
\end{tabular}

Table 2. Comparison of ground state energies of atoms' ground-state energies in hartrees (htr) with atom number $Z$ : $2 \leq Z \leq 30$, between experimental values and Bohr's energies. When $Z$ is small, there is a better agreement, and the trend is basically sound. But none of Bohr's energies are within the chemical accuracy of 5 decimal places.

We now provide several examples below to illustrate stable as well as unstable electron configurations according to Bohr's model.

Example 1 (Stable electron configurations for the ground state of the Bohr atom model, with $Z: 3 \leq Z \leq 10)$. We set up the problem as follows. In order to avoid the 3 -D rotational congruence as stated in Theorem 1, we fix the direction of $\boldsymbol{r}_{1}$ along the positive $z$-axis and that of $\boldsymbol{r}_{2}$ on the $(x, z)$-plane, as follows:

$$
\begin{cases}\boldsymbol{r}_{1}=r_{1} \boldsymbol{k}, & \\ \boldsymbol{r}_{2}=r_{2} \sin \theta_{2} \boldsymbol{i}+r_{2} \cos \theta_{2} \boldsymbol{k}, & \\ \boldsymbol{r}_{i}=r_{i} \sin \theta_{i} \cos \phi_{i} \boldsymbol{i}+r_{i} \sin \theta_{i} \sin \phi_{i} \boldsymbol{j}+r_{i} \cos \theta_{i} \boldsymbol{k}, & i \geq 3 \\ 0 \leq \theta_{i} \leq \pi, \quad 0 \leq \phi_{i} \leq 2 \pi, & i \geq 2\end{cases}
$$

Then the relative distances become

$$
\left\{\begin{array}{lll}
r_{1 i}=\left(r_{1}^{2}-r_{i}^{2}-2 r_{1} r_{i} \cos \theta_{i}\right)^{1 / 2}, & & 2 \leq i \leq Z, \\
r_{2 i}=\left[r_{2}^{2}+r_{i}^{2}-2 r_{2} r_{i}\left(\sin \theta_{2} \sin \theta_{i} \cos \phi_{i}+\cos \theta_{2} \cos \phi_{i}\right)\right]^{1 / 2}, & & 3 \leq i \leq Z, \\
r_{i j}=\left[r_{i}^{2}+r_{j}^{2}-2 r_{i} r_{j}\left(\sin \theta_{i} \sin \theta_{j} \cos \left(\phi_{i}-\phi_{j}\right)+\cos \theta_{i} \cos \theta_{j}\right)\right]^{1 / 2}, & & 3 \leq i<j<Z .
\end{array}\right.
$$

The stable electron configurations corresponding to minimal ground state energy of (1.5) are shown in the next few figures, along with the data given in the captions. 


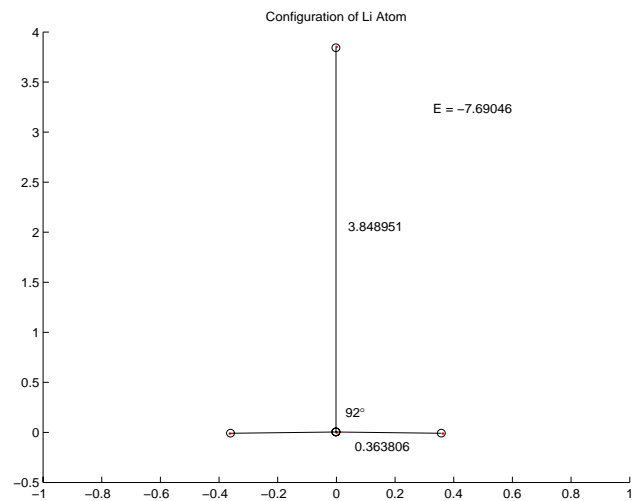

Fig. 2 For lithium (Li), $Z=3$. The electron configuration is shown above. We have

$$
\begin{aligned}
& r_{1}=r_{2}=0.363806, \quad r_{3}=3.848951, \\
& \theta_{2}=1.605660=91.99754143^{\circ} \neq \frac{\pi}{2}, \\
& \theta_{3}=1.605660=91.99754131^{\circ} \neq \frac{\pi}{2}, \phi_{3}=\pi, \\
& E=-7.69046(h t r) ; h t r=\text { hartree. }
\end{aligned}
$$

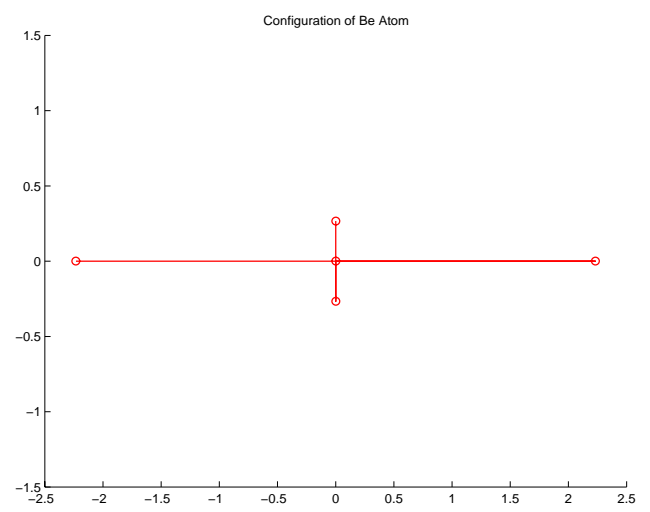

Fig. 3 For beryllium (Be), $Z=4$. We have

$$
\begin{aligned}
& r_{1}=r_{2}=0.26690, \theta_{2}=3.14159=\pi, \\
& r_{3}=r_{4}=2.23196, \theta_{3}=\theta_{4}=1.57080=\frac{\pi}{2}, \phi_{3}=\phi_{4}=3.14159=\pi, \\
& E=-14.84035(h t r) .
\end{aligned}
$$




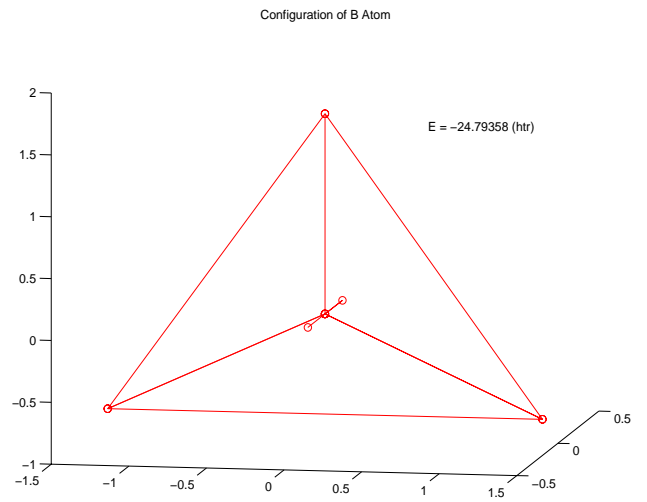

Fig. 4 For boron (B), $Z=5$. We have

$$
\begin{aligned}
& r_{1}=r_{2}=0.21081, r_{3}=r_{4}=r_{5}=1.61777, \\
& \theta_{2}=2.09440=\frac{2 \pi}{3}, \theta_{3}=2.09440=\frac{2 \pi}{3}, \phi_{3}=3.14159=\pi, \\
& \theta_{4}=1.57080=\frac{\pi}{2}, \phi_{4}=1.57080=\frac{\pi}{2} \\
& \theta_{5}=1.57080=\frac{\pi}{2}, \phi_{5}=-1.57080=-\frac{\pi}{2}, \\
& E=-24.79358(\mathrm{htr}) .
\end{aligned}
$$

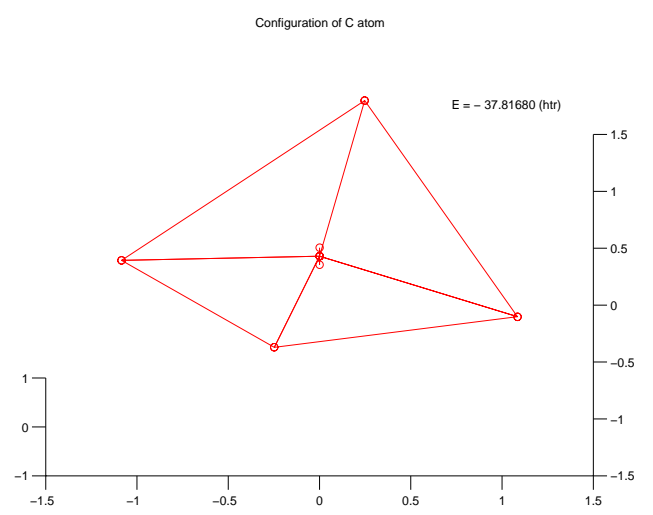

Fig. 5 For carbon (C), $Z=6$. We have

$$
\begin{aligned}
r_{1} & =r_{2}=0.17398, r_{3}=r_{4}=r_{5}=r_{6}=1.29353, \theta_{2}=3.14159=\pi, \\
\theta_{3} & =2.10638, \phi_{3}=-0.22466, \\
\theta_{4} & =1.03522, \phi_{4}=1.34614, \\
\theta_{5} & =2.10638, \phi_{5}=2.91693, \\
\theta_{6} & =1.03522, \phi_{6}=-1.79546, \\
E & =-37.81680(h t r) .
\end{aligned}
$$




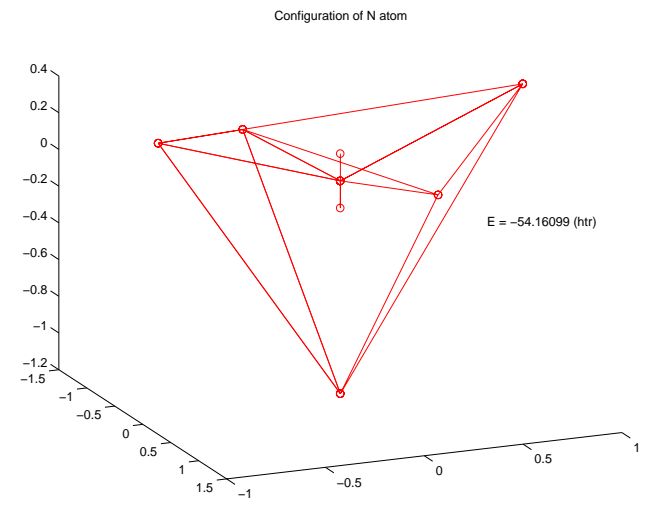

Fig. 6 For nitrogen (N), $Z=7$. We have

$$
\begin{aligned}
r_{1} & =r_{2}=0.14822, r_{3}=r_{6}=1.06095, r_{4}=r_{5}=1.05175, r_{7}=1.15887 \\
\theta_{2} & =3.14159=\pi \\
\theta_{3} & =-\theta_{6}=1.21801, \phi_{3}=\phi_{6}=-0.19528 \\
\theta_{4} & =\theta_{5}=1.47408, \phi_{4}=1.37551 \\
\phi_{5} & =-1.76608 \\
\theta_{7} & =3.14159=\pi, \phi_{7}=0.32885 \\
E & =-54.16099(\text { htr })
\end{aligned}
$$

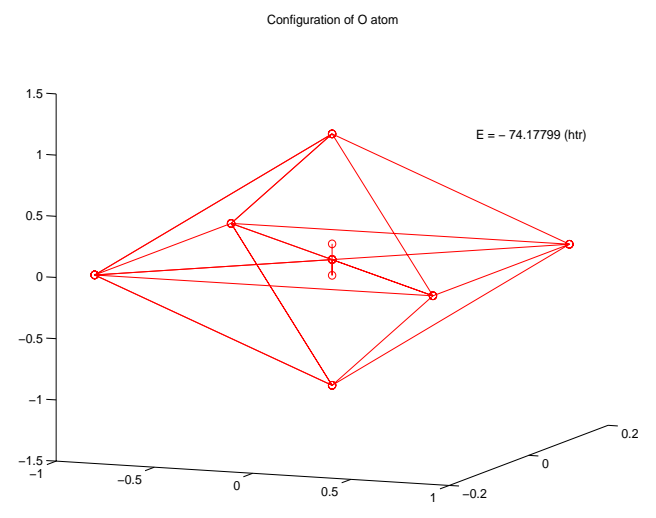

Fig. 7 For oxygen $(\mathrm{O}), Z=8$. We have

$$
\begin{aligned}
r_{1} & =r_{2}=0.12910, r_{3}=r_{4}=r_{5}=r_{6}=0.87770, r_{7}=r_{8}=1.02674 \\
\theta_{2} & =\theta_{8}=\pi, \theta_{3}=\theta_{4}=\theta_{5}=\theta_{6}=\pi / 2, \theta_{7}=0 \\
\phi_{3} & =-0.19720, \phi_{4}=1.37360, \phi_{5}=2.94440, \phi_{6}=-1.76800, \phi_{7}=-0.48203, \phi_{8}=-0.73723, \\
E & =-74.17799(h t r) .
\end{aligned}
$$




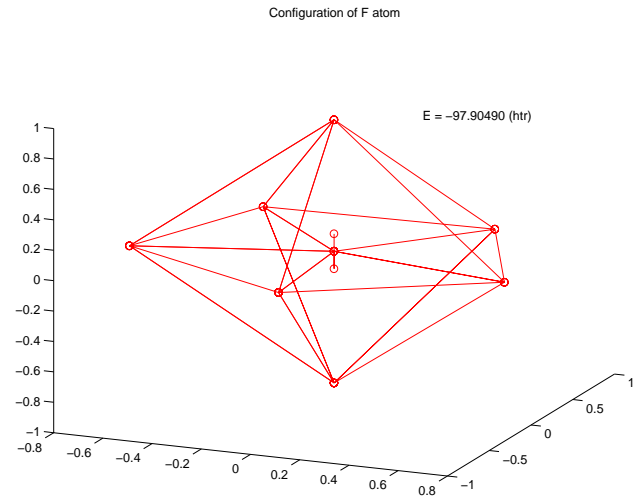

Fig. 8 For fluorine (F), $Z=9$. We have

$$
\begin{aligned}
r_{1} & =r_{2}=0.11435, r_{3}=r_{4}=r_{5}=r_{6}=r_{7}=0.78823, r_{8}=r_{9}=0.86558, \\
\theta_{2} & =\theta_{9}=\pi, \theta_{3}=\theta_{4}=\theta_{5}=\theta_{6}=\theta_{7}=\pi / 2, \theta_{8}=0, \\
\phi_{3} & =-0.26152, \phi_{4}=0.99512, \phi_{5}=2.25176, \phi_{6}=3.50839, \phi_{7}=4.76503, \phi_{8}=-0.21356, \\
\phi_{9} & =-0.02196, \\
E & =-97.90490(\mathrm{htr}) .
\end{aligned}
$$

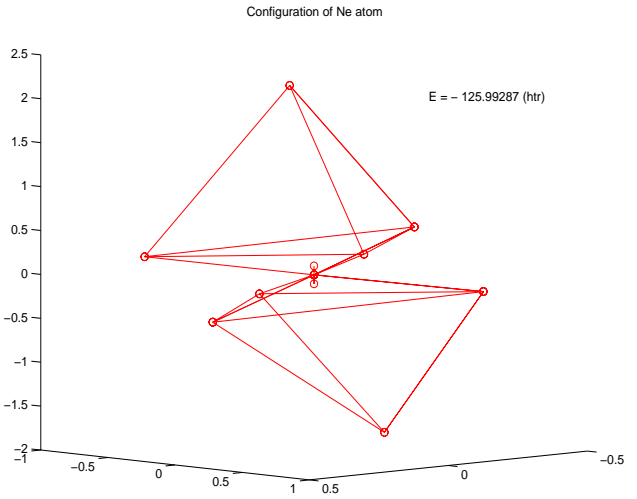

Fig. 9 For neon (NE), $Z=10$. We have

$$
\begin{aligned}
r_{1} & =r_{2}=0.10263, r_{3}=2.14617, r_{4}=0.63726, r_{5}=0.63288, r_{6}=0.63387, \\
r_{7} & =0.63355, r_{8}=0.63008, r_{9}=0.62496, r_{10}=1.87083, \\
\theta_{2} & =3.13987(\neq \pi), \theta_{3}=-0.06317, \theta_{4}=0.99093, \theta_{5}=1.06946, \theta_{6}=0.98591, \theta_{7}=2.13822, \\
\theta_{8} & =2.11486, \theta_{9}=2.04094, \theta_{10}=3.01675, \\
\phi_{3} & =-0.16895, \phi_{4}=0.14127, \phi_{5}=2.17293, \phi_{6}=4.25346, \phi_{7}=1.11058, \phi_{8}=3.25805, \\
\phi_{9} & =-0.94826, \phi_{10}=-1.32532, \\
E & =-125.99287(\text { htr }) .
\end{aligned}
$$

Example 2 (Saddle-type unstable colinear electron configurations, $Z=3$ ). Consider the case of $\mathrm{Li}$, $Z=3$. Write

$$
\left\{\begin{array}{l}
\boldsymbol{r}_{3}=r_{3} \boldsymbol{k}, \boldsymbol{r}_{1}=r_{1} \sin \theta_{1} \boldsymbol{i}+r_{1} \cos \theta_{1} \boldsymbol{k} \\
\boldsymbol{r}_{2}=r_{2} \cos \theta_{2} \boldsymbol{k}+r_{2} \sin \theta_{2} \cos \phi_{2} \boldsymbol{i}+r_{2} \sin \theta_{2} \sin \phi_{2} \boldsymbol{j}
\end{array}\right.
$$


We consider

$$
\min _{\boldsymbol{R} \in \mathbb{R}_{z}^{9}} E(\boldsymbol{R}), \quad \boldsymbol{R}=\left(\boldsymbol{r}_{1}, \boldsymbol{r}_{2}, \boldsymbol{r}_{3}\right)=\left(\left[\begin{array}{c}
0 \\
0 \\
r_{1}
\end{array}\right],\left[\begin{array}{c}
0 \\
0 \\
r_{2}
\end{array}\right],\left[\begin{array}{c}
0 \\
0 \\
r_{3}
\end{array}\right]\right),
$$

i.e., all 3 vectors $\boldsymbol{r}_{1}, \boldsymbol{r}_{2}$ and $\boldsymbol{r}_{3}$ are required to be colinear on the $z$-axis. This can be done by setting $\phi_{2}=\pi$ and by requiring $\theta_{1}$ and $\theta_{2}$ to be equal to either 0 or $\pi$ in (5.3).

For problem (5.4), we have found 5 critical points $\boldsymbol{R}_{i}^{\dagger}, i=1,2,3,4,5$, with respect to the 3 scalar variables $r_{1}, r_{2}$ and $r_{3}$. See Table 3 below.

\begin{tabular}{|c|c|c|c|c|c|c|}
\hline \multirow{2}{*}{ critical points } & \multicolumn{4}{|c|}{ coordinates (cf. (5.3)) (set $\phi_{2}=\pi$ ) } & \multirow{2}{*}{ Energies } \\
\cline { 2 - 6 } & $r_{1}$ & $r_{2}$ & $r_{3}$ & $\theta_{1}$ & $\theta_{2}$ & \\
\hline $\boldsymbol{R}_{1}^{\dagger}$ & 1.0334 & 0.3133 & 7.2778 & 0 & 0 & -5.5985 \\
\hline $\boldsymbol{R}_{2}^{\dagger}$ & 0.3622 & 0.3647 & 4.1865 & 0 & $\pi$ & -7.6837 \\
\hline $\boldsymbol{R}_{3}^{\dagger}$ & 0.3647 & 0.3622 & 4.1865 & $\pi$ & 0 & -7.6837 \\
\hline $\boldsymbol{R}_{4}^{\dagger}$ & 1.0967 & 0.3174 & 2.2462 & $\pi$ & $\pi$ & -5.7752 \\
\hline $\boldsymbol{R}_{5}^{\dagger}$ & 0.3174 & 1.0967 & 2.2462 & $\pi$ & $\pi$ & -5.7752 \\
\hline
\end{tabular}

Table 3 Locations of four critical points of problem (5.4). Note that only $\boldsymbol{R}_{2}^{\dagger}$ and $\boldsymbol{R}_{3}^{\dagger}$ are true minimizers for (5.4), while $\boldsymbol{R}_{1}^{\dagger}, \boldsymbol{R}_{4}^{\dagger}$ and $\boldsymbol{R}_{5}^{\dagger}$ are just local minima for (5.4).

Note that by Theorem 4 , all these points $\boldsymbol{R}_{i}^{\dagger}, i=1,2,3,4,5$, are critical points of $E$ on $\mathbb{R}^{3 Z}$. On the other hand, fix $\phi_{2}=\pi$ only. Then $\boldsymbol{r}_{1}, \boldsymbol{r}_{2}$ and $\boldsymbol{r}_{3}$ are coplanar and

$$
\begin{aligned}
E\left(\boldsymbol{r}_{1}, \boldsymbol{r}_{2}, \boldsymbol{r}_{3}\right) & =\frac{1}{2 r_{1}}+\frac{1}{2 r_{2}}+\frac{4}{2 r_{3}}-\sum_{i=1}^{3} \frac{3}{r_{i}}+\left(\frac{1}{\left|\boldsymbol{r}_{1}-\boldsymbol{r}_{2}\right|}+\frac{1}{\left|\boldsymbol{r}_{1}-\boldsymbol{r}_{3}\right|}+\frac{1}{\left|\boldsymbol{r}_{2}-\boldsymbol{r}_{3}\right|}\right) \\
& \equiv E\left(r_{1}, r_{2}, r_{3}, \theta_{1}, \theta_{2}\right) .
\end{aligned}
$$

Numerical computations of the Hessian matrix $H=\left[\partial^{2} E / \partial \theta_{i} \partial \theta_{j}\right]_{1 \leq i, j \leq 2}$ and Lemma 1 show that, indeed, these $\boldsymbol{R}_{i}^{\dagger}$ are saddle-type critical points. See Table 4.

\begin{tabular}{|c|c|c|}
\hline point & two eigenvalues of Hessian $\left[\partial^{2} E / \partial \theta_{i} \partial \theta_{j}\right]$ & eigenvectors \\
\hline $\boldsymbol{R}_{1}^{\dagger}$ & $-1.7530,-0.0187$ & $(-0.7120,-0.7022)^{T},(0.7022,-0.7120)^{T}$ \\
\hline $\boldsymbol{R}_{2}^{\dagger}$ & $-0.0061,0.6831$ & $(-0.7290,0.685)^{T},(0.6845,0.7290)^{T}$ \\
\hline $\boldsymbol{R}_{3}^{\dagger}$ & $-0.0061,0.6831$ & $(0.6845,-0.7290)^{T},(-0.7290,-0.6845)^{T}$ \\
\hline $\boldsymbol{R}_{4}^{\dagger}$ & $-1.4170,0.0542$ & $(0.7014,0.7128)^{T},(-0.7128,0.7014)^{T}$ \\
\hline $\boldsymbol{R}_{5}^{\dagger}$ & $-1.4170,0.0542$ & $(0.7014,0.7128)^{T},(-0.7128,0.7014)$ \\
\hline
\end{tabular}

Table 4 Eigenvalues of the Hessian with respect to the angular variables $\theta_{1}$ and $\theta_{2}$.

In Fig. 10, we plot the energy surfaces of $E$ with respect to the angular variables $\theta_{1}$ and $\theta_{2}$ in a neighborhood of these critical points $\boldsymbol{R}_{i}^{\dagger}$. 

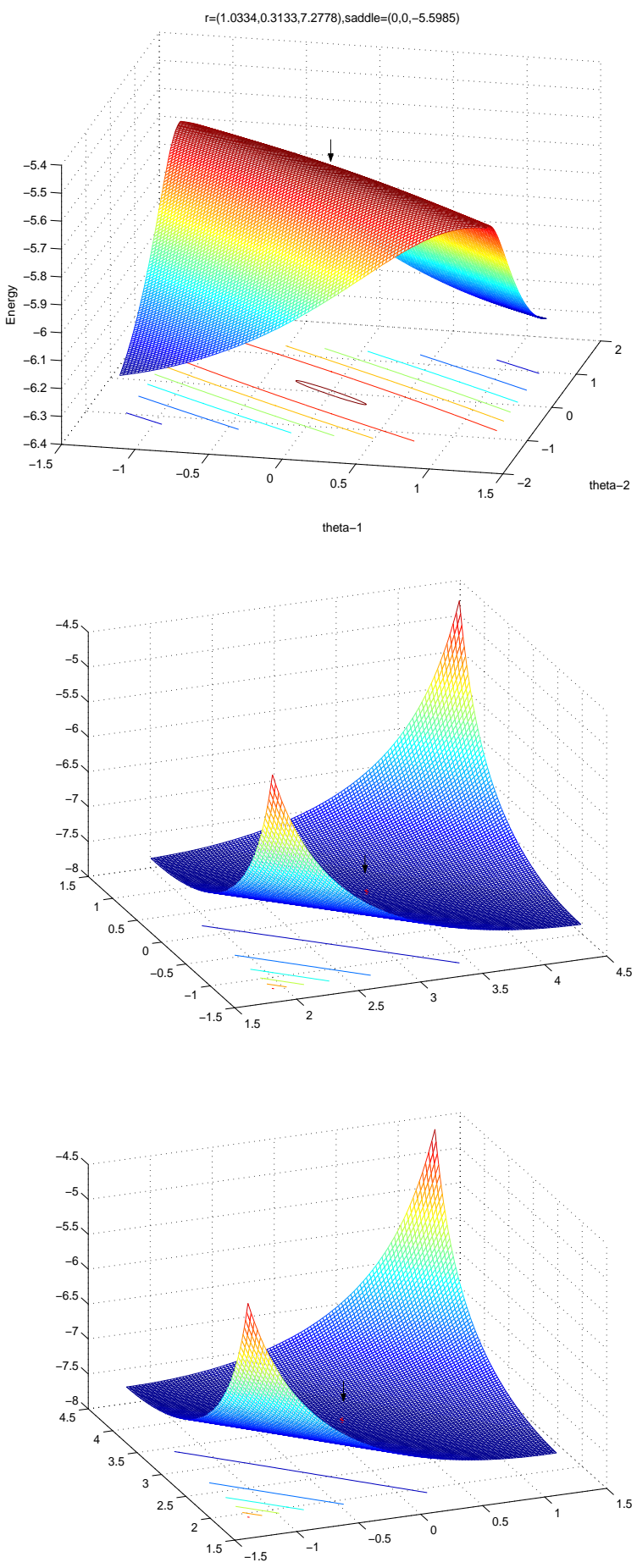


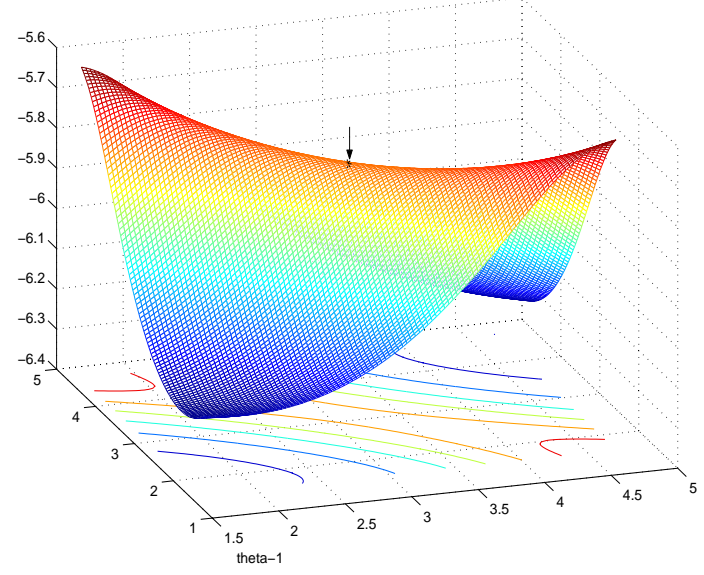

$r=(1.0967,0.3174,2.2462)$,saddle=(pi, pi, -5.7752$)$

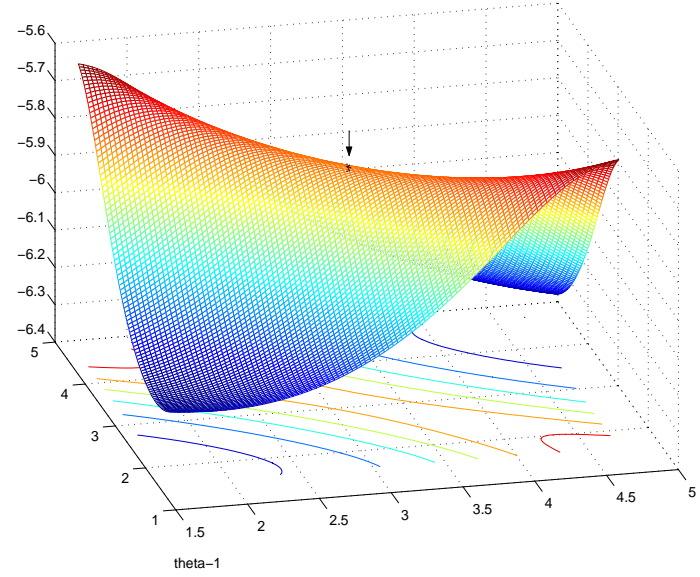

Fig. 10 The energy surface $E$ plotted against the angular variables $\theta_{1}$ and $\theta_{2}$ (cf. (5.3)) near the critical points $\boldsymbol{R}_{i}^{\dagger}, i=1,2,3,4,5$, (cf. Table 3 ) in sequential order, whose locations are pin-pointed by an arrow. These surfaces are all of the saddle type.

It is quite interesting to note from Fig. 2 earlier that the global minimum value of $E$ on $\mathbb{R}^{9}$ is $-7.69046 \mathrm{htr}$, while the global minimum value of $E$ in (5.4) by restricting all $\boldsymbol{r}_{i}, i=1,2,3$, to lie on the $z$-axis is $-7.6837 \mathrm{htr}$, (cf. Table 3), which differs from -7.69046 by less than $0.1 \%$.

Example 3 (Saddle type unstable coplanar electron configurations, $Z=5$ ). In order to find unstable coplanar electron configurations, $Z$ must be greater than or equal to 5 , as Section 4 has proved that $Z=3$ can only have stable configurations and Fig. 2 has provided a numerical evidence that $Z=4$ also has stable coplanar electron configurations. For $Z=5$, we apply Theorem 6 to find coplanar configurations, which must be of the saddle-type and unstable, as Fig. 4 shows that a stable configuration cannot be coplanar. Several unstable configurations can be seen in Fig. 11. 

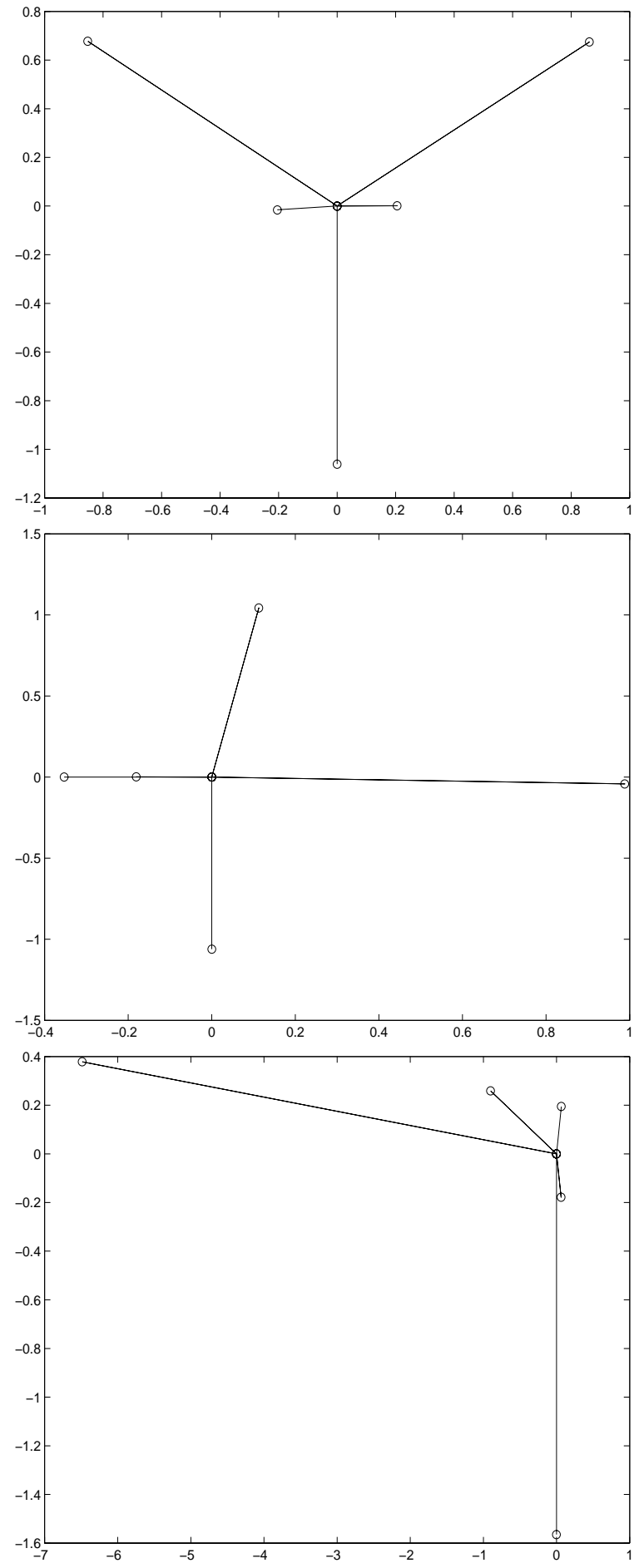

Fig. 11 For $Z=5$ (boron), the above three configurations are obtained according to Theorem 6 by finding critical points of $E$ on the $(x, y)$-plane numerically. They should be contrasted with the one in Fig. 4. Coordinates of the five vectors, as well as the corresponding energy values, given 
from top to bottom for these three configurations, are:

$$
\begin{aligned}
& (-0.2045,-0.0156,0),(0.2050,0.0014,0),(0.8625,0.6741,0),(-0.8524,0.6777,0),(0 .,-1.0604,0) \\
& \quad \text { with } E_{\min }=-28.9146 \\
& (-0.3532,0,0),(-0.1808,0.0011,0),(0.9884,-0.0419,0),(0.1128,1.0034,0),(0 .,-1.0610,0) \\
& \quad \text { with } E_{\min }=-24.9439 \\
& (0.0661,0.1942,0),(0.0628,-0.1781,0),(-6.4876,0.3784,0),(-0.9024,0.2594,0),(0 .,-1.5647,0) \\
& \quad \text { with } E_{\min }=-27.5713
\end{aligned}
$$

\section{Conclusions}

In this paper, we have conducted basic mathematical analysis for existence of minimal energy configurations and certain properties of critical points for the Bohr energy function. Relevant numerical results are also developed and presented.

There are still interesting problems remaining open. One among them is the coplanarity of the stable electron configuration for $Z=4$, which we have not yet been able to prove in Section 4 . Also, the determination of the many symmetries manifested in Figs. 2-9 has not been achieved.

Bohr's original model for molecules [3] (which generalized the atomic case studied here) had some difficulties which has recently been improved by Svidzinsky, Scully and Hershbach [13]. It has many interesting mathematical features therein worth investigation and we hope to be able to do it in the near future.

Acknowledgement. This research is supported in part by ONR Grants (N00014-03-1-0693 and N00014-04-1-0336), NSF Grant DMS 0311905 and a TITF initiative from Texas A\&M University. We also thank Drs. Siu A. Chin and Anatoly Svidzinsky for helpful discussions.

\section{References}

[1] N. Bohr, On the constitution of atoms and molecules, Part I, Phil. Magz. 26 (1913), 1-25.

[2] — ibid., Part II, Systems containing only a single nucleus, Phil. Magz. 26 (1913), 476.

[3] , ibid., Part III, Systems containing several nuclei, Phil. Magz. 26 (1913), 857875 .

[4] S.A. Chin, Personal communications, Dept. of Physics, Texas A\&M Univ., College Station, TX, 2004.

[5] R.W. Gomez, Ground and excited energy levels of helium-like atoms using a simple geometrical model, Eur. J. Phys. 13 (1992), 135-138.

[6] R.D. Harcourt, Bohr orbit theory revisited:I. Ground state energies for the helium isoelectronic sequence, J. Phys. B 16(1983), 2647-2657.

[7] R.D. Harcourt, Bohr orbit theory revisited:II. Energies for 1S, 2P, 3D and 4F states of helium, Int. J. Quantum Chem. 31(1987), 445-453. 
[8] D.R. Hershbach, J. Avery and O. Goscinski (eds.), Dimensional Scaling in Chemical Physics, Kluwer, Dordrecht, The Netherlands, 1992.

[9] M.J.M. Hill, On functions of more than two variables analogous to tesseral harmonics, Transactions of the Cambridge Philos. Soc. 13 (1883), p. 36.

[10] R.D. Harcourt, J. Beckworth and R. Feigin, Simple "local" energy studies on the origin of Hund's rule of maximum spin multiplicity and the energies of $1 s n l_{\max }$ configurations for He, $\mathrm{Ne}^{8+}$, and $\mathrm{AR}^{16+}$, Chem. Phys. Lett. 26 (1974), 126-130.

[11] K. Patton, Chemistry models learning outline, http://www.lionden.com/chemistry_models.htm

[12] A.A. Svidzinsky, M.O. Scully, and D.R. Herschbach, Simple and surprisingly accurate approach to the chemical bond obtained from dimensionality scaling, Phys. Rev. Lett. 95 (2005), 080401.

[13] A.A. Svidzinsky, M.O. Scully, and D.R. Herschbach, Bohr's 1913 molecular model revisited, Proc. Natl Acad. Sci. 102 (2005), 11985-11988.

[14] G. Tanner, K. Richter and J.-M. Rost, The theory of two-electron atoms: between ground state and complete fragmentation, Rev. Mod. Phys. 72 (2000), 497-544.

[15] A. Trabesinger, 'Bohr'n again, Nature Physics Published online: 25 August 2005, http://www.nature.com/nphys/journal/vaop/nprelaunch/full/nphys115.html 